\title{
Potent inhibitors of toxic alpha-synuclein identified via cellular time-resolved FRET biosensors
}

Anthony R. Braun (iD), Elly E. Liao (D)', Mian Horvath ${ }^{2}$, Prakriti Kalra ${ }^{3}$, Karen Acosta (iD), Malaney C. Young ${ }^{4}$, Noah Nathan Kochen (iD), Chih Hung Lo ${ }^{1}$, Roland Brown ${ }^{5}$, Michael D. Evans (iD) , William C. K. Pomerantz (iD ${ }^{3}$, Elizabeth Rhoades ${ }^{4,6}$, Kelvin Luk (iD ${ }^{2}$, Razvan L. Cornea $\mathbb{I D}^{7,8}$, David D. Thomas ${ }^{7,8}$ and Jonathan N. Sachs $\mathbb{i D}^{1 凶}$

We have developed a high-throughput drug discovery platform, measuring fluorescence resonance energy transfer (FRET) with fluorescent alpha-synuclein (aSN) biosensors, to detect spontaneous pre-fibrillar oligomers in living cells. Our two aSN FRET biosensors provide complementary insight into aSN oligomerization and conformation in order to improve the success of drug discovery campaigns for the treatment of Parkinson's disease. We measure FRET by fluorescence lifetime, rather than traditional fluorescence intensity, providing a structural readout with greater resolution and precision. This facilitates identification of compounds that cause subtle but significant conformational changes in the ensemble of oligomeric states that are easily missed using intensity-based FRET. We screened a 1280-compound small-molecule library and identified 21 compounds that changed the lifetime by $>5$ SD. Two of these compounds have nanomolar potency in protecting SH-SY5Y cells from aSN-induced death, providing a nearly tenfold improvement over known inhibitors. We tested the efficacy of several compounds in a primary mouse neuron assay of aSN pathology (phosphorylation of mouse aSN pre-formed fibrils) and show rescue of pathology for two of them. These hits were further characterized with biophysical and biochemical assays to explore potential mechanisms of action. In vitro aSN oligomerization, single-molecule FRET, and protein-observed fluorine NMR experiments demonstrate that these compounds modulate aSN oligomers but not monomers. Subsequent aggregation assays further show that these compounds also deter or block aSN fibril assembly.

npj Parkinson's Disease (2021)7:52; https://doi.org/10.1038/s41531-021-00195-6

\section{INTRODUCTION}

Preventing or reversing the pathological misfolding and selfassociation of a-synuclein (aSN) can rescue a broad spectrum of pathological cellular insults that manifest in Parkinson's disease (PD), dementia with Lewy bodies, multiple systems atrophy, and other alpha-synucleinopathies ${ }^{1-9}$. Over the past several years, the field's understanding of which forms of aSN assemblies are most toxic has evolved, shifting away from mature fibrils rich in $\beta$-sheets toward structurally heterogeneous, early-stage oligomers ${ }^{9-14}$. Many questions remain regarding these heterogeneous oligomers, including what their exact macromolecular constituency is in cells (i.e., what co-mingles with aSN ${ }^{1,15-17}$ ), whether they possess any well-defined structural motifs-various forms, including a tetramer, have been described in the literature ${ }^{18-24}$-and how variations in their molecular properties contribute to toxicity. These unknowns complicate drug discovery, where it remains to be seen whether a unique small-molecule binding site or epitope even exists, as has been suggested recently for fibrils ${ }^{25-27}$. More generally, it is unclear whether direct binding of an inhibitor to aSN oligomers, or fibrils for that matter, is more efficient than indirectly targeting the cellular machinery and pathways that can prevent or alter their assembly ${ }^{28}$.

Despite many unknowns, there is clear therapeutic potential in shifting the equilibrium distribution of aSN monomers, oligomers, and fibrils $4,26,27,29-36$. Unfortunately, it has proven experimentally challenging to either control the stochasticity and heterogeneity of in vitro oligomerization of purified aSN or to directly monitor pre-fibrillar oligomers in cells. Consequently, most discovery campaigns have targeted in vitro fibril growth. A recent study that used thioflavin-T (ThT) as the engine for a high-throughput screening (HTS) campaign identified an inhibitor of purified aSN fibrillization that reduced inclusions in human neuroglioma $(\mathrm{H} 4)$ cells $^{27}$. Nevertheless, even at $10 \mu \mathrm{M}$, the inhibitor was only effective in $50 \%$ of cells ${ }^{27}$. The same inhibitor also protected neuron loss in a Caenorhabditis elegans model of PD but again in less than half the worms ${ }^{27}$. Another recent HTS study used Förster or fluorescence resonance energy transfer (FRET) to monitor sodium dodecyl sulfoxide (SDS)-induced fibrillization of recombinant $\mathrm{aSN}^{37}$. Hits identified in that study predominantly targeted monomeric aSN (aSN $(m))$, with the best hit compounds rescuing oligodendrocytes from exogenously added aSN aggregateinduced cell death, albeit at high compound concentrations $(30 \mu \mathrm{M})^{37}$. While targeting aSN fibrils has shown promise, concerns persist as to whether these inhibitors of purified aSN fibrils translate to the more complex assemblies (e.g., non-fibrillar oligomers) formed in cells and if they can be cytoprotective in the low nanomolar to picomolar range (as antibodies are ${ }^{38-40}$ ).

Anle138b is a particularly prominent example of a smallmolecule inhibitor of aSN oligomerization (with an apparent $\mathrm{EC}_{50}$ of $2.8 \mu \mathrm{M}$ ). Anle138b prevents the formation of iron-induced aSN aggregates in vitro and shows strong positive effects in mouse models of $\mathrm{PD}^{41}$. In our studies below, we show that Anle138b

\footnotetext{
'Department of Biomedical Engineering, University of Minnesota, Minneapolis, MN, USA. ${ }^{2}$ Department of Pathology and Laboratory Medicine, University of Pennsylvania, Philadelphia, PA, USA. ${ }^{3}$ Department of Chemistry, University of Minnesota, Minneapolis, MN, USA. ${ }^{4}$ Biochemistry \& Molecular Biophysics Graduate Group, University of

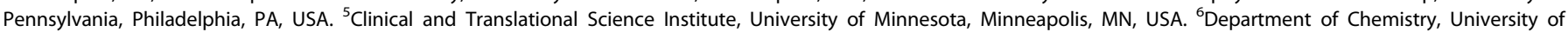
Pennsylvania, Philadelphia, PA, USA. 'Department of Biochemistry, Molecular Biology and Biophysics, University of Minnesota, Minneapolis, MN, USA. ${ }^{8}$ Photonic Pharma LLC, Minneapolis, MN, USA. ${ }^{\bowtie}$ email: jnsachs@umn.edu
} 
protects $\mathrm{SH}-\mathrm{SY} 5 \mathrm{Y}$ cells from aSN-induced death with an $\mathrm{EC}_{50}$ of $\approx 900 \mathrm{nM}$. A more recent study reached nanomolar potency $(500 \mathrm{nM})$ using engineered peptides that mimic fibril structures and prevented the growth of pre-formed fibril (PFF) seeds with a single $25 \mu \mathrm{M}$ inhibitor dose required for cytoprotection ${ }^{25}$.

There is one recent study that has resulted in small-molecule inhibitors of non-fibrillar aggregates. In a technological feat, bimolecular fluorescence complementation (BiFC) was used to monitor aSN oligomerization in a cellular HTS campaign, yielding small molecules that protected $\mathrm{H} 4$ cells in the mid-nanomolar range (reported $\left.I C_{50} \approx 500 \mathrm{nM}\right)^{42}$. Here we adopt a variation on this same theme by engineering FRET-based biosensors that monitor aSN oligomers in cells without requiring direct reporter protein interaction and maturation of the fluorophore, which is required in BiFC. Our aSN FRET cellular biosensor screening platforms are engineered to target toxic, early-stage, spontaneous aSN oligomers and conformations. Using single mEGFP and TagRFP fusion constructs (inter-protomeric FRET) and doublefusion constructs (intra-protomeric FRET), these two biosensors monitor oligomerization and conformation by using fluorescence lifetimes (FLTs). A key advantage of our approach is the detection of FLTs rather than the traditional fluorescence intensities. This increases the precision of the measurements by a factor of thirty ${ }^{43}$, enabling detection of minute structural changes within the ensemble of aSN assemblies and thereby the discovery of small molecules that may otherwise be missed with traditional measurements.

The inter-protomeric and double-fusion (intra-protomeric) FRET biosensors were transiently transfected into HEK293 cells and HTS were conducted on the 1280-compound Library of Pharmacologically Active Compounds (LOPAC) to validate our screening platforms. Together, these two biosensors provide complementary insight into aSN oligomerization and conformation. We then tested the efficacy of hit compounds in SH-SY5Y cells overexpressing unlabeled wild-type (WT) aSN (to rule out labeling artifacts), and we report that two small molecules, Demeclocycline $\mathrm{HCl}$ (DEM) and Ro 90-7501 (RO), completely protected SH-SY5Y cells from aSN-induced death. The compounds are active in the low nanomolar range $\left(\mathrm{EC}_{50}=65\right.$ and $78 \mathrm{nM}$, respectively) and are an order of magnitude more potent than previously described small molecules. We tested the efficacy of these compounds in a primary mouse neuron assay of aSN pathology (phosphorylation of mouse aSN PFFs) and show rescue for DEM (but not RO), as well as another lead compound (( \pm )-Bay K 8644, BAY) that was not effective in the SH-SY5Y cell models. Given the very different nature of this murine model from the screening platform, it is notable to have found effective compounds.

We further characterized these three hits with a combination of biochemical and biophysical assays to determine potential mechanism of action (MOA) for the rescue of aSN-induced pathology. Using recombinant protein oligomerization and fibrillization assays coupled to single-molecular FRET (smFRET) and protein-observed fluorine (PrOF) nuclear magnetic resonance (NMR) experiments, we confirmed that these compounds directly interact with aSN oligomers, increasing the extent of oligomerization and shifting the equilibrium toward non-toxic, off-pathway aSN oligomers. Subsequent seeded ThT assays demonstrate that these compounds also deter or block fibril assembly (at a substoichiometric ratio to $\operatorname{aSN}(\mathrm{m}))$, further demonstrating the complex interplay between pre-fibrillar oligomers, beta-sheet fibrils, and cell death.

Our inter- and intra-protomeric FRET aSN biosensors provide a new platform for evaluating aSN conformation and oligomerization. Our HTS screens using these FRET aSN biosensors identified new hit compounds that target aSN pathology at nanomolar potencies and validated multiple compounds that were identified in previous screens using other HTS platforms. We further demonstrate the ability of our drug-discovery platform to identify hit compounds that can target a multitude of therapeutic MOAs, which further demonstrates the broad potential application of our cellular aSN oligomer FRET biosensors.

\section{RESULTS}

\section{aSN biosensor engineering}

The cellular aSN biosensors developed here monitor spontaneous oligomerization and the ensemble of conformations of aSN (Supplementary Fig. 1). Each aSN construct is fused with either donor (N-terminal mEGFP) or acceptor (C-terminal TagRFP) or both (mEGFP-aSN-TagRFP), where all XFPs are monomerized forms ${ }^{44}$. The HTS strategy employed here uses HEK293 cells and a fluorescence lifetime plate reader (FLT-PR) platform designed to monitor specific protein interactions and structural changes in living cells ${ }^{43,45,46}$. FLT detection increases the precision of FRETbased screening by a factor of 30 compared with conventional fluorescence intensity detection ${ }^{43}$ and provides exquisite sensitivity to resolve minute structural changes within protein ensembles. The improved signal to noise is due to FLT being an intrinsic property of the XFP and not sensitive to fluctuations in steady-state fluorescence intensity. This sensitivity, along with our series of aSN-XFP fusion constructs, allows for the monitoring of both inter-protomeric aSN interactions and oligomeric conformations.

Optimization of expression conditions of FLT biosensor was determined by transiently transfecting a titration of donor-toacceptor (D:A) ratios into HEK293 cells. Cells were harvested after $48 \mathrm{~h}$ of transfection and then plated and read in the FLT-PR. Figure 1a presents the FLT of each condition with the corresponding FRET in Fig. 1b. We observe a decrease in FLT (i.e., increased FRET) up to a plateau that transitions at D:A of 1:8. The magnitudes of the measured lifetimes (and hence FRET, Figs. 1a, b and 3) are slightly higher than observed for our recent tau biosensor ${ }^{47}$ and roughly consistent with other biosensors using this FLT platform ${ }^{43,45-52}$. Figure 3 shows the results from the intra-protomeric, double-fusion biosensor, which for WT aSN had a slightly lower, though still substantial, FRET signal.

We next characterized the relative fluorescence contribution of donor and acceptor aSN constructs using a spectral unmixing plate reader (SUPR), which records the entire emission spectra. Subsequent data analysis resolves the contributions from the four primary spectral components: (1) donor fluorescence, (2) acceptor fluorescence, (3) cellular autofluorescence background, and (4) Raman scattering. Supplementary Fig. 2 highlights control spectra and Supplementary Fig. 3 presents the spectra from the titration in Fig. 1a. Quantification of the spectral unmixing fits is presented in Fig. 1c. As expected, we observe a clear increase in the acceptor fluorescence relative to the donor at higher DNA titrations.

The SUPR experiment provides further guidance in the selection of optimal biosensor conditions. As the amount of donor DNA decreases, the fraction of donor fluorescence diminishes, reaching similar levels to the background cellular autofluorescence (green vs. gray wedges, Fig. 1c). As background fluorescence becomes more significant, we observe a decreased signal-to-noise ratio, resulting in the observed increased variability in the FLT signal.

We observed a similar trend in expression levels via flow cytometric analysis of aSN inter- and intra-protomeric cellular biosensors (Supplementary Fig. 4). For the inter-protomeric system, there is a strong acceptor signal that continues to accumulate with increasing titration. For the intra-protomeric biosensor, the 1:1 expression profile is clearly visible as the strong diagonal in the second quadrant. The results are further supported with western blot analysis of biosensor cell lysates from the D:A titration (Fig. 1d), which illustrates distinct bands for both GFP-aSN and aSN-RFP constructs (see Supplementary Fig. 5a for loading control for Fig. 1d). 

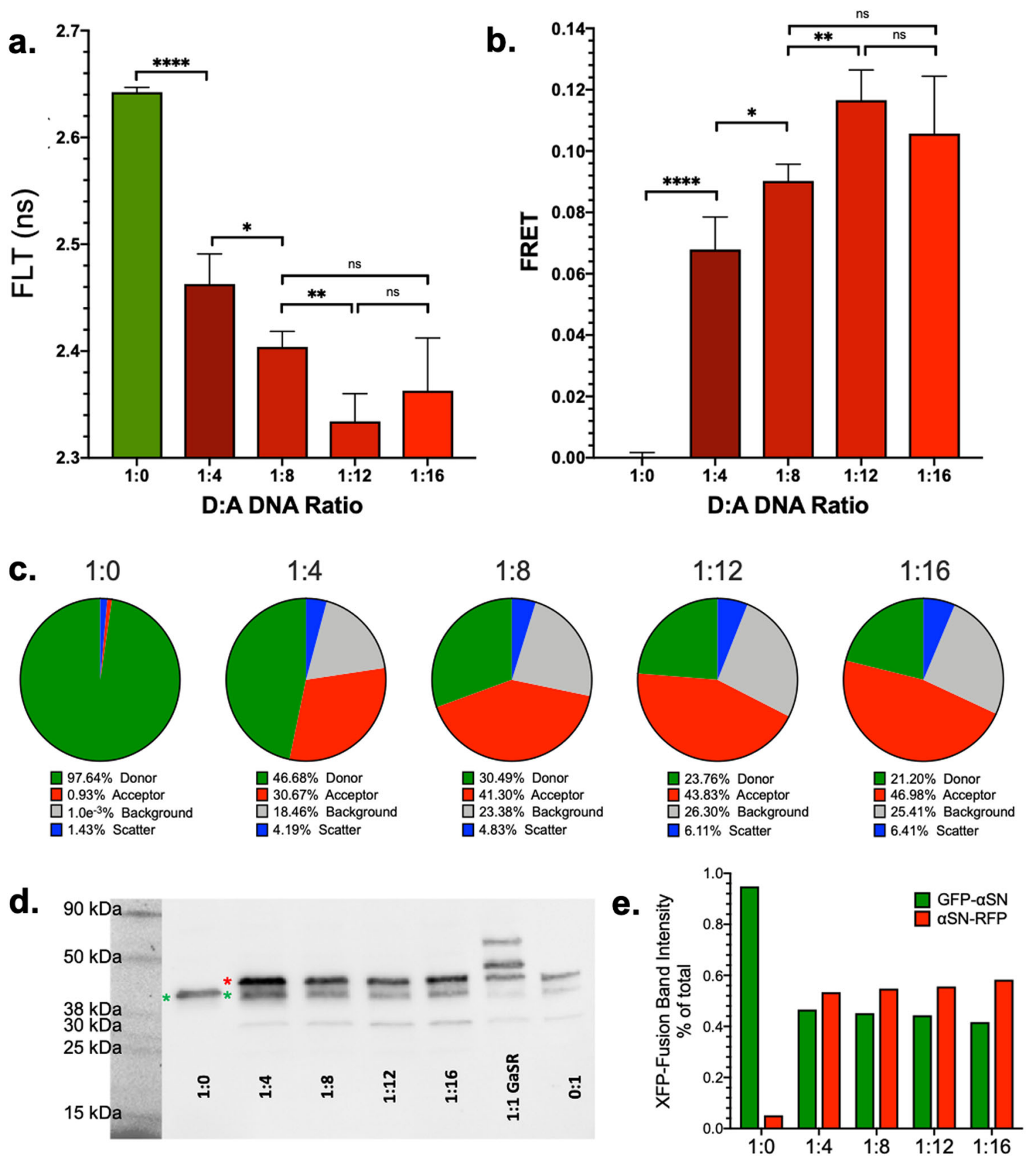

Fig. 1 Engineering of aSN cellular FRET biosensors. a Fluorescence lifetime (FLT) measured with the FLT-PR for a range of donor and acceptor (D:A) ratios show significant reduction of FLT that saturates around D:A of 1:8. b FRET determined at each D:A ratio in a. c Spectral unmixing of each biosensor illustrates the shift in ratio of D:A throughout the increasing D:A DNA titration (see Supplementary Fig. 3 for source spectra). The relative donor-to-background signal (gray to green) is directly correlated to the degree of variance for each system. d Immunoblot for $\alpha \mathrm{SN}$ in WT $\alpha \mathrm{SN}$ oligomer (inter-protomeric) biosensor and intra-protomeric biosensor. Cell lysates for each D:A ratio were analyzed via SDS-PAGE gel and probed for $\alpha$ SN using the Syn 1 antibody. All samples were transfected with a constant total DNA (15 $\mu g$ DNA for $10 \mathrm{~cm}$ plate). GFP- $\alpha \mathrm{SN}$ manifests as a strong band at $\sim 40 \mathrm{kD}$ in the 1:0 lane. The C-terminal RFP- $\alpha \mathrm{SN}$ fusion runs slightly higher than the GFP- $\alpha$ SN construct. Similarly, C-terminal truncation is observed for $\alpha$ SN-RFP. Note: RFP truncation does not negatively affect our FRET assay as we only monitor the FLT for the donor (GFP construct). Supplementary Fig. 6 explores $\alpha \mathrm{SN}$ truncation in more depth via comparisons of total $\alpha \mathrm{SN}$ (Syn1) and LB509-positive immunoblots. e Densitometric analysis of the two most prominent XFP bands (indicated by green and red asterisks $\left(^{*}\right)$ in $\mathbf{d}$ shows an increase in acceptor expression, albeit at a reduced rate relative to D:A DNA titration. Experiments were performed with $n=3 ;{ }^{*} p<0.05,{ }^{* *} p<0.01,{ }^{* * *} p<0.0001$, ns indicates not significant as determined by Student's $t$ test.

In addition to full-length aSN fusion constructs, we observe both C-terminally and N-terminally truncated aSN species. These were confirmed by western blot analysis of total aSN (Syn1) ${ }^{53}$ vs. full-length aSN (LB509) ${ }^{54}$ along with green fluorescent protein (GFP) and red fluorescent protein (RFP) immunoreactivity (Supplementary Fig. 6). This effect is more observable in cells expressing the GFP-aSN-RFP and aSN-RFP fusion where the Cterminal truncation (resulting in Syn1-positive but not RFP-positive bands) is more prominent than N-terminal (Syn1-positive, LB509positive, GFP-negative bands). The presence of aSN truncation products biased toward C-terminal truncation motivated the use of an N-terminal fused donor. In these FRET biosensors, any 
a.

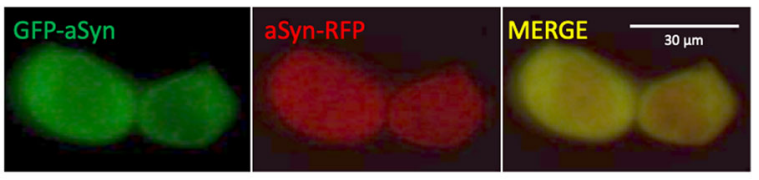

b.

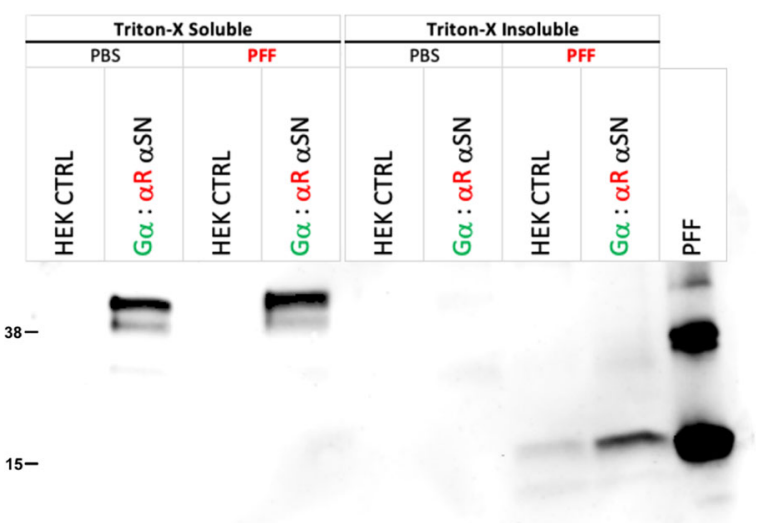

Fig. 2 Characterization of aSN cellular FRET biosensors. a Fluorescence live cell imaging of our inter-protomeric cellular FRET biosensors co-expressing GFP- $\alpha \mathrm{SN} / \alpha \mathrm{SN}$-RFP constructs illustrate a diffuse expression profile (non-punctate). b To explore whether our biosensor FRET stems from soluble oligomers or

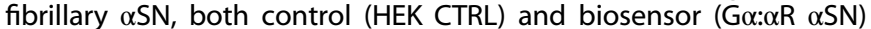
transfected HEK cells were treated with PFFs (400 ng) or vehicle (PBS) for $24 \mathrm{~h}$. Immunoblots of Triton-X soluble and insoluble with $2 \%$ SDS extraction were probed with Syn 1 antibody and showed that all biosensor fusion constructs were extracted in the soluble fraction for both PBS and PFF conditions, indicating that soluble protomer/oligomeric $\alpha S N$ is present in our $\alpha \mathrm{SN}$ biosensors. From the blots, insoluble $\alpha \mathrm{SN}$ derived from PFF is detected in PFF-treated TX100 insoluble fraction of cell lysate. See Supplementary Fig. 5 for loading control.

protein cleavage that results in free donor fragments would have a similar FLT signal as soluble GFP, reducing the observed FRET signal window. In contrast, due to the abundance of expression, free acceptor XFP fragments do not negatively impact FRET to the same extent, as only donor FLT is monitored.

The conclusion of these assays narrowed the selection of a D:A DNA ratio of 1:8, which optimized the FLT/FRET signal window and minimizes FLT signal variability.

\section{aSN biosensor characterization}

Our aSN FRET cellular biosensor screening platforms are engineered to target toxic, spontaneous aSN oligomers and conformations. It is therefore important to characterize the types of protein-protein assemblies present in our cellular biosensor. Fluorescence microscopy (Fig. 2a), western blot analysis (Fig. 2b and Supplementary Figs. 6-8), and cell-seeded ThT aggregation assays (Supplementary Fig. 9) provide insight into some of the direct protein-protein interactions present and were used to verify that our biosensors are monitoring early-stage aSN oligomers, and not fibrils. Figure 2a shows cells co-transfected with GFP-aSN/aSNRFP, where the expression pattern is notably diffuse, devoid of puncta indicative of aggregated (fibrillar) aSN.

We then explored whether the FRET observed in our biosensors is derived from soluble oligomers or fibrillar aSN by treating control (CTRL) and biosensor (Ga:aR aSN) transfected HEK cells with PFF or vehicle (phosphate-buffered saline (PBS)) for $24 \mathrm{~h}$. Since we are targeting pre-fibrillar oligomers, we focused on shorter transfection and treatment regimes. Previous studies have shown that fibrillar aSN aggregates accumulate in detergent- insoluble fractions ${ }^{55-59}$, especially when induced by PFF seeds. Immunoblot analyses of Triton-X soluble and insoluble (with $2 \%$ SDS extraction) fractions showed that all the biosensor fusion constructs were extracted in the soluble fraction for both PBS and PFF treatment, indicating that soluble protomer/oligomeric aSN is present in our aSN biosensors. The experimental conditions for our aSN biosensor HTS are more comparable to PBS treatment; as seen in (Fig. 2b), there is minimal insoluble/fibrillar aSN in the PBStreated samples (loading control for Fig. 2b presented in Supplementary Fig. 5b). Only treatment with PFF resulted in insoluble aSN in the Triton- $X$ insoluble fraction, where control cellular lysates showed only a modest increase in the amount of detergent-insoluble $\mathrm{aSN}(\mathrm{m})$ and Ga:aR aSN cells exhibited a threefold increase in the amount of Triton-X-insoluble aSN $(\mathrm{m})$ over controls (Fig. 2b). This shows that PFF addition is required for aSN recruitment into the insoluble fractions. Our short PFF treatment regimen is within the 2-4-day recruitment lag phase, where we observed a lack of recruitment of aSN biosensor constructs into PFF-induced puncta, as seen in other studies ${ }^{58,59}$.

We next determined whether our cellular aSN biosensor are phosphorylated at Serine-129 $\left(\mathrm{pS}^{129}\right)$. Accumulation of $\mathrm{pS}^{129}$ is a hallmark of PD pathology. Supplementary Fig. 7 presents immunoblot analysis of biosensor cell lysates probed for $\mathrm{pS}^{129}$ (left) or total aSN (right) with unlabeled aSN and aSN PFF used as control samples for positive $\mathrm{pS}^{129}$ staining. We observed strong $\mathrm{pS}^{129}$ reactivity with the unlabeled and GFP-aSN sample; however, both inter- and intra-protomeric biosensors have a greatly reduced $\mathrm{ps}^{129}$ signal. This suggests that our current biosensor platform is predominantly aSN assemblies without $\mathrm{pS}^{129}$.

The robust FRET signal in the inter-protomeric biosensor suggests that direct aSN-aSN interaction is present in the biosensor cells. Using immunoprecipitation (IP) (Supplementary Fig. 8), we isolated soluble GFP or GFP-aSN from HEK293 cell homogenates and probed for the pull-down of aSN or RFP to indicate direct aSN-aSN interactions or XFP-induced artifacts, respectively. Supplementary Fig. 8 shows that GFP-aSN is able to pull down endogenous unlabeled aSN while lysate from control cells and cells expressing soluble GFP or RFP do not. Although these IP experiments were not conclusive in resolving direct protein-protein interaction between GFP-aSN and aSN-RFP fusion constructs in the inter-protomeric biosensor, our results do show that non-specific interactions between soluble GFP and aSN are not observed either. Due to the early stage of aSN assemblies in these biosensors, it is plausible that the protein-protein interactions which are responsible for FRET are not sufficiently mature to survive the IP assay conditions.

Next we explored whether or not the inter-protomeric aSN biosensor constructs contain seeding-competent aSN assemblies. Supplementary Fig. 9 presents cell lysate-seeded ThT aggregation assay results for a series of HEK293 cell lysate conditions (untransfected, unlabeled aSN, and inter-protomeric biosensor) with and without recombinant aSN or PFF seed. Indeed, only samples that had both recombinant $a \mathrm{SN}(\mathrm{m})$ and PFF seed displayed positive ThT fluorescence. The biosensor-expressing lysate was indistinguishable from control HEK cell lysate results. This confirms that the aSN assemblies responsible for producing FRET are not seeding competent and therefore are pre-fibrillar in nature. Taken together, these results provide evidence that our FLT aSN biosensor experiments are conducted in conditions that do not promote fibrillization of aSN unless induced with aSN PFFs.

\section{aSN biosensor sensitivity}

As a first test of the sensitivity of this platform, we also developed a set of mutant A53T aSN biosensors. The A53T familial mutant is known to increase aggregation propensity and toxicity ${ }^{60-64}$. Comparison of WT and A53T inter- and intra-protomeric biosensors demonstrate significant FLT and FRET differences 

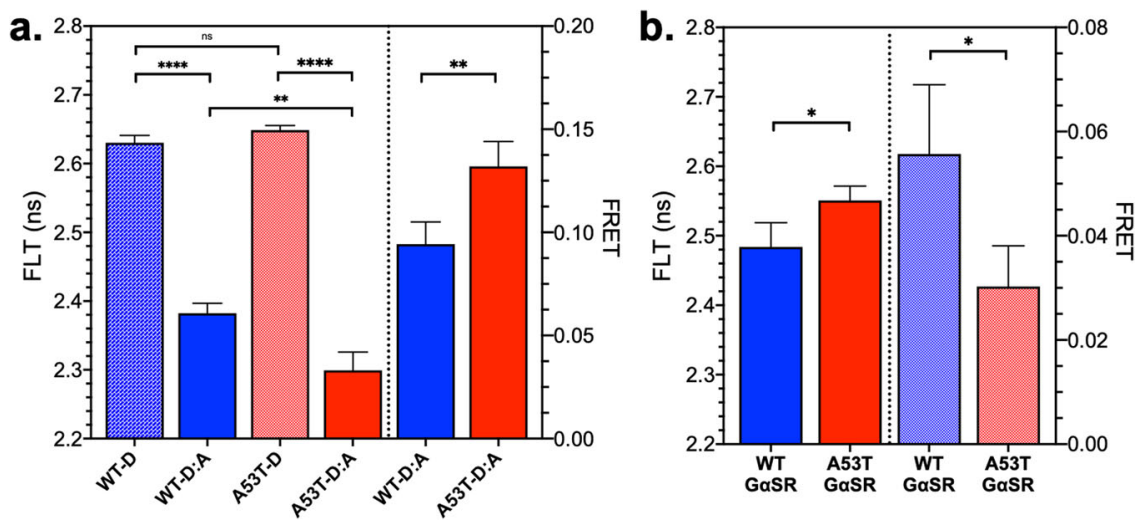

Fig. 3 Characterization of aSN cellular FRET biosensors. a Comparison of WT and A53T $\alpha$ SN inter-protomeric FRET biosensors show decreased fluorescence lifetime and corresponding increased FRET for the aggregation-prone A53T construct relative to WT. $\mathbf{b}$ Comparison of WT and A53T $\alpha$ SN intra-protomeric FRET biosensors shows an increased fluorescence lifetime and decrease in FRET for the A53T relative to WT. Experiments were done with $n=5 ;{ }^{*} p<0.05,{ }^{* *} p<0.01,{ }^{* * * *} p<0.0001$, ns indicates no significant difference as determined by Student's $t$ test.

(Fig. 3a, b, respectively). As expected, the aggregation-prone A53T mutant resulted in increased FRET relative to WT for the interprotomeric biosensor. This agrees with previous findings by Tosatto et al. that cell-free aggregation of A53T aSN oligomerizes more rapidly than WT and preferentially matures into a more compact, $\beta$-sheet-positive oligomer with increased interprotomeric $\mathrm{FRET}^{65}$. Interestingly, in the intra-protomeric biosensor, the mutant induced a reduction in FRET, suggesting a more extended conformation for A53T, highlighting the sensitivity of these biosensors to changes in aSN conformation in these complex, heterogeneous assemblies.

We next looked into the relative D:A expression levels for both WT and A53T biosensor systems. Although the intrinsic nature of FLT is a major advantage for FLT-FRET biosensors, during their development it is essential to characterize biosensor expression for an aggregation-prone protein like aSN because more aSN can drive increased aggregation. Using SUPR experiments on both WT and A53T inter-protomeric biosensor, we observed a consistent and significant increase in D:A ratio for A53T relative to WT (Supplementary Fig. 10a-c). This complicates the interpretation of the increased FRET in A53T, as it may be due to differences in expression as well as increased aggregation propensity. However, comparison of SUPR fits from the intra-protomeric biosensors (Supplementary Fig. 10d-f) revealed that both WT and A53T have similar expression profiles. The intra-protomeric construct ensures a 1:1 D:A ratio, improving consistency across biosensor variants, with XFP folding and maturation being primarily responsible for fluorescence differences. Even with the SUPR discrepancies in the inter-protomeric biosensor comparison, these experiments do show that our technology is sensitive to small changes in aSN sequence and confirms the capacity to monitor structural changes in an intrinsically disordered protein system.

The addition of XFP fusion proteins to any cellular system can induce artifacts and changes in the target proteins' native function. To further evaluate our biosensors' sensitivity, we compared the aSN inter- and intra-protomeric biosensors to a series of control biosensors to investigate the potential for nonspecific interactions being driven by the XFP fusion. Figure 4 presents a comparison of the aSN inter-protomeric (D:A 1:8) to a series of control biosensors, including soluble GFP/RFP (D:A 1:8), GFP-aSN to soluble RFP, GFP-aSN to TNFR1-RFP, and GFP to aSNRFP. We observe a consistent FRET efficiency of $\sim 0.02$ for control GFP/RFP (D:A 1:8) biosensor system with no significant differences for other non-specific FRET biosensors. A comparison of intraprotomeric aSN and control biosensor is presented in Supplementary Fig. 11. The significantly larger FRET from our inter- protomeric GFP-aSN:aSN-RFP system provides a large aSNdependent FRET signal window for use in our HTS campaigns.

\section{Pilot HTS with the LOPAC library for the oligomer and monomer conformation aSN FRET biosensors}

Prior to each pilot HTS, FLT and emission spectrum were measured to verify signal level, basal FRET, coefficient of variance (CV), and similarity index. For each HTS, cells were dispensed into compound plates-1536-well $(10 \mu \mathrm{L} /$ well)-and incubated with the compounds $(10 \mu \mathrm{M})$ or dimethyl sulfoxide (DMSO; $1 \%)$ for $90 \mathrm{~min}$. FLT measurements were acquired with the FLT-PR. After deconvolving the instrument response function, a single exponential fit was used to determine the FLT for both the aSN cellular FRET biosensor $\left(\tau_{\mathrm{DA}}\right)$ and the donor-only control biosensor $\left(\tau_{\mathrm{D}}\right)$. FRET efficiency was determined by Eq. 1. CV was determined from control plates ( $N=384$ wells).

A major challenge to any fluorescence-based HTS platform stems from optically interfering (e.g., fluorescent) compounds in the library. Fluorescent compounds (FC) were flagged using a spectral similarity index filter, which evaluates the ratio of intensity from two bandwidths that span the donor emission spectrum and are excluded as potential false positives ${ }^{45,46,49,51,66}$. FLT values for all compounds that passed the FC filter are presented in Fig. 5 for both the inter-protomeric (Fig. 5a) and intra-protomeric (Fig. 5b) biosensor HTS. Each compound plate included 256 DMSO control wells as a control for the aSN FRET biosensor signal quality over time. Both biosensor HTS datasets exhibited a tight Gaussian distribution of compounds (Supplementary Fig. 12). Compounds that changed FLT by $>5$ standard deviations (SD) were considered hits (Fig. 5, red data points). The 5-SD threshold is an arbitrary definition that was made to reduce the number of compounds that are investigated in subsequent secondary assays. In total, 21 hits were explored, 19 hits from the inter-protomeric screen and 8 hits from the intra-protomeric biosensor at the 5-SD threshold (with 6 overlapping compounds identified in both screens, see Supplementary Table 1).

The screen identified multiple compounds within the LOPAC library that were previously known to attenuate aSN-induced toxicity: AGK2 (a SIRT2 antagonist) and Bio (an autophagy inducer) ${ }^{67,68}$, as well as those shown via BiFC (e.g., DEM, PD173952, PD169316) ${ }^{42}$. DEM was not pursued as a functional inhibitor in the BiFC study due to the potential interference of the tetracycline derivative compound with their Tet-Off expression system. Additional hits in our screen span a range of known pharmacological functions that directly affect cellular pathways known to be disrupted by aSN overexpression (e.g., modulating 
6

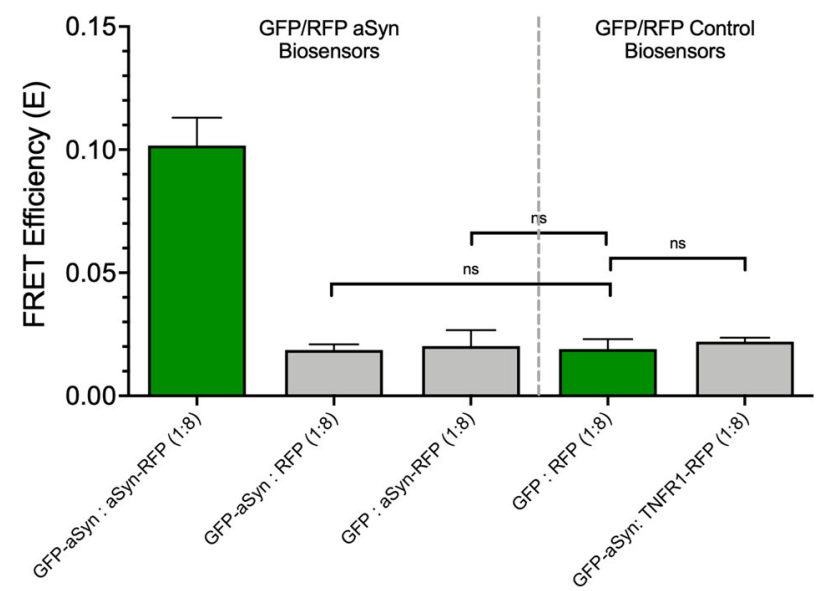

Fig. 4 XFP fusion does not contribute significantly to background FRET signal. One of the major concerns with any tagged or fusion protein assay are the potential of non-specific protein-protein interactions being driven via the XFP or fluorescent tag. Here we compared our inter-protomeric $\alpha \mathrm{SN}$ cellular FRET biosensor with soluble mEGFP/TagRFP at the same D:A ratio as well as heterobiosensor combinations to explore potential non- $\alpha \mathrm{SN}$-driven FRET. GFP- $\alpha$ SN to soluble RFP, soluble GFP to $\alpha S N-R F P$, and GFP- $\alpha S N$ to TNFR1-RFP systems demonstrate little to no difference from soluble GFP/RFP systems. We conclude that the significant FRET in the interprotomeric biosensor relative to soluble GFP/RFP is therefor due to direct $\alpha \mathrm{SN}-\alpha \mathrm{SN}$ interactions. ns indicates no significant difference as determined by Student's $t$ test.

oxidative stress, reducing inflammation, inhibiting nitric oxide synthase, and upregulating autophagy). The identification of known effectors of aSN pathology as well as compounds relevant to aSN-induced cellular dysfunction reinforce the efficacy, sensitivity, and selectivity of our aSN cellular FRET biosensor.

FLT changes in both inter- and intra-protomeric biosensors may indicate of a wide range of conformational changes (Supplementary Fig. 1). An increase in the basal inter-protomeric FLT (decreased FRET) could result from the dissociation of spontaneous formed oligomers or through structural rearrangement of oligomers that shift the ensemble of donor-to-acceptor XFP distances further apart. Similarly, a reduction in FLT (increased FRET) could be due to increased oligomerization (e.g., a shift in the equilibrium between monomer and soluble, non-toxic tetramer $^{19,24}$ ) or structural rearrangement toward a more compact oligomer. The intra-protomeric double-fusion biosensor provides complementary insight into aSN, as FLT changes can arise from changes in oligomerization or in monomer conformation, where reduced FLT corresponds to a more compact ensemble of conformations.

\section{FLT dose response of hits with WT and A53T aSN FRET biosensors}

Hits from the pilot HTS of the LOPAC library were selected and dispensed into 384-well plates to verify reproducible and specific FLT trends using the WT and A53T oligomer (inter-protomeric) FRET biosensors. Figure 6 presents a subset of these doseresponse curves for hit compounds RO, BAY, SB206553, and DEM, along with known control compound EGCG. For both WT and A53T biosensors, these compounds displayed similar doseresponse signal. Concentrations above $50 \mu \mathrm{M}$ showed significant cytotoxicity for some compounds, prohibiting an accurate FLT IC $C_{50}$ determination. Of the hits tested, only SB206553 showed an increasing FLT trend, suggesting dissociation or loosening of monomers within oligomers (decreased FRET). All other hits (including control compound EGCG) displayed a reduced donor
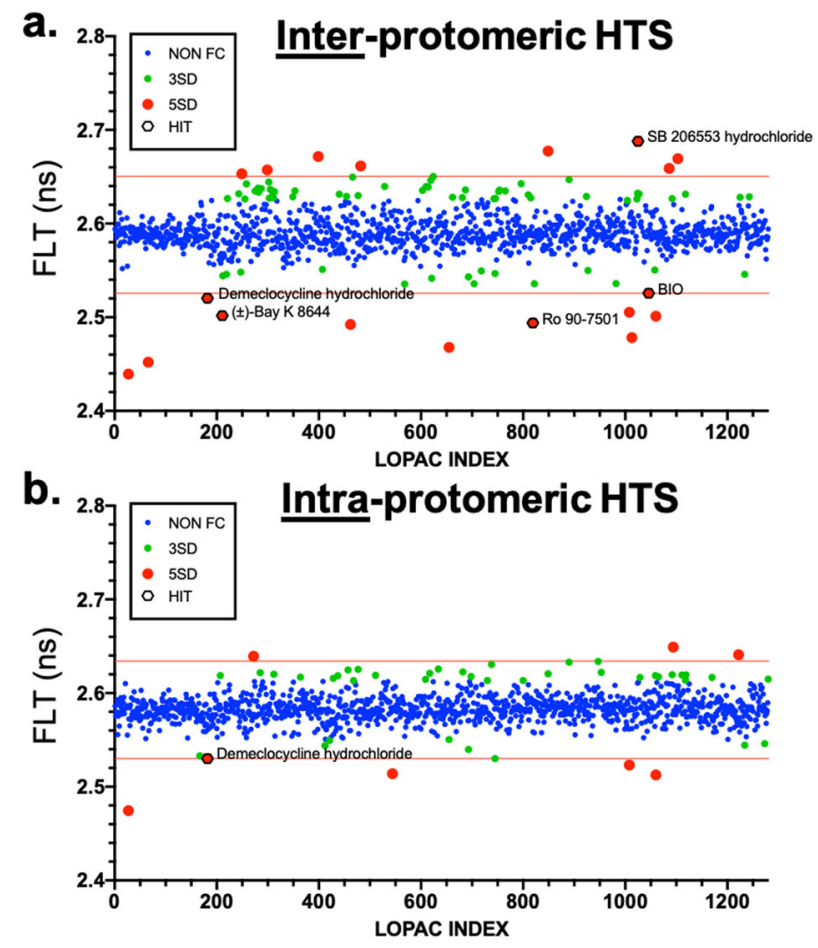

Fig. 5 Pilot HTS on cellular aSN FRET biosensors. a, b Screens of the 1280-compound LOPAC library were performed in a single 1536well plate for both the inter-protomer (a) and intra-protomeric (b) biosensor. Pilot HTS provided 19 and 8 hits, respectively, that changed FLT by $>5$ SD (red). For some systems, a 3-SD (red+green) threshold is sufficient if secondary assays can process the increased throughput.

FLT (increased FRET), indicative of increased oligomer formation and/or remodeling of oligomers into a more compact conformation. Assay quality was determined by $Z^{\prime}$ (Eq. 2) where a $Z^{\prime}>0.5$ indicates good assay quality. Using EGCG as control compound and the hit DEM, we calculated $Z^{\prime}$ value of 0.76 and 0.66 , respectively, indicating excellent assay quality.

Hits were also evaluated with the intra-protomeric aSN and control, non-specific biosensors. Figure 7 illustrates that our hit compounds induced a significant change in FRET at $10 \mu \mathrm{M}$ in the intra-protomeric aSN biosensor. The compounds RO, BAY, and DEM as well as the control EGCG each reduced FRET, indicating either reduction in oligomerization or expanded oligomer conformation. Tests of these compounds in two control biosensor systems (GFP/RFP at 1:8 and GFP-linker32-RFP; see Supplementary Fig. 13a, b, respectively) show no significant FRET change. These control biosensors provide support that the hit compounds have an aSN-dependent effect and do not modulate the XFPs directly. Interpreting these results in light of the inter-protomeric concentration-response curve (CRC) suggests that these hit compounds are changing the oligomer structure and not dissociating oligomer assemblies.

\section{Secondary assay: total cellular viability}

We note the significant potential for artifacts due to the labeling of aSN with large synthetic fluorophores (fusion constructs add $\sim 25 \mathrm{kDa}$ of XFP onto a $15 \mathrm{kDa}$ protein). Thus, all secondary cellular assays presented below (cytotoxicity, expression, pathology) are done with unlabeled aSN. It is also important to note that we cannot determine, a priori, whether an increase or decrease in FLT correlates to reduced toxicity. 


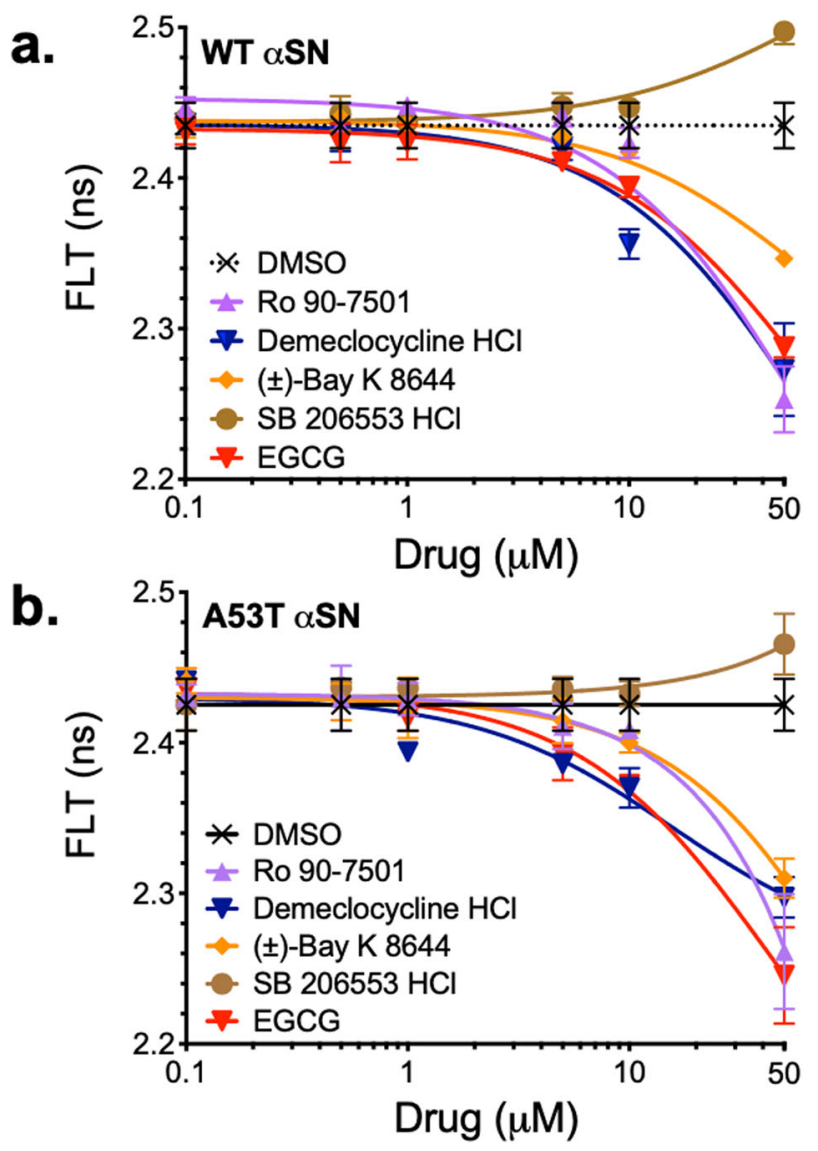

Fig. 6 FRET dose response of pilot HTS hits. A subset of hits were evaluated using both WT (a) and A53T (b) oligomer biosensor to verify both increased FLT (disrupting $\alpha \mathrm{SN}-\alpha \mathrm{SN}$ interactions) and decreased FLT (tighter aggregation or remodeled oligomers). Three hits that reduced FLT (RO, BAY, DEM) demonstrated similar response as control EGCG, whereas hit SB206553 demonstrated mild recovery of FLT, suggesting dissociation.

Hit compounds were evaluated for rescue of aSN-induced cytotoxicity in using an SH-SY5Y neuroblastoma cell model of alpha-synucleinopathy ${ }^{18,69-72}$. Overexpression of WT aSN showed significant reduction in cell viability ( $46 \%$ total viability, Fig. 8 a and Supplementary Fig. 14). There is clear dose-dependent rescue of toxicity for hits RO and DEM, with reduced effect for BAY. Although hit SB206553 demonstrated reproducible and dosedependent increased FLT (Fig. 7), there was no observed effect on cytotoxicity, highlighting that a change in FLT does not necessarily correlate with changes in toxicity. A seven-point CRC for RO, BAY, DEM, and Anle138b (positive control compound ${ }^{41,73}$ ) was performed to determine $\mathrm{IC}_{50}$ for each compound. Both $\mathrm{RO}$ and DEM demonstrated potent inhibition of aSN-induced toxicity with an $\mathrm{IC}_{50}$ of 78 and $65 \mathrm{nM}$, respectively (Fig. 8a).

We note that these $I C_{50}$ values, which are an order of magnitude more potent than previously published small-molecule inhibitors, are significantly lower than the lifetime dose responses. There are several possible explanations for this: they are performed in different cell lines (HEK293 vs. SH-SY5Y), under different treatment conditions (FRET is measured after a 48-h transfection and 20-90 min compound exposure, whereas cytotoxicity assays are conducted with a 72-h transfection and 48-h exposure to the compound.), with different constructs (+/- FRET labels) and with different phenomena being measured. (FRET measures changes in distance with an $R^{-6}$ dependence, with picosecond FLT resolution vs. cytotoxicity, which measures bulk lactate dehydrogenase enzyme released in media relative to total cell via luminescence

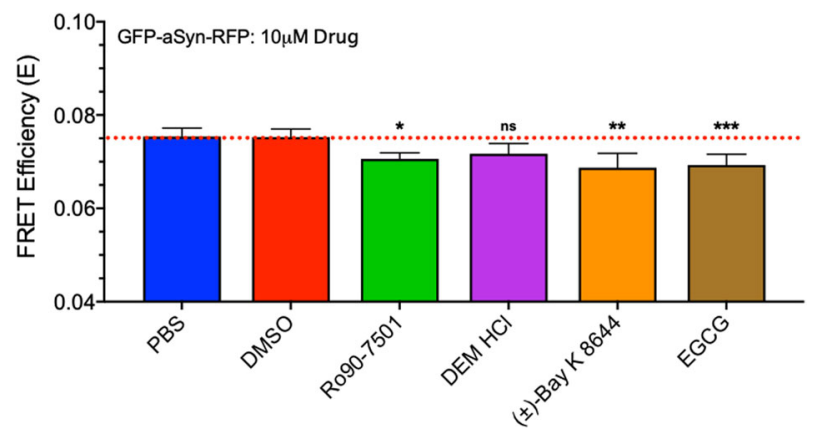

Fig. 7 FRET response to pilot HTS hits on intra-protomeric aSN biosensor. Hits that demonstrated robust, reproducible FRET response in the inter-protomeric biosensor CRC were then evaluated with the intra-protomeric $\alpha \mathrm{SN}$ biosensor. With both donor and acceptor XFP fused to one construct, this biosensor is sensitive to both oligomer formation as well as conformation. Three hits reduced the intra-protomeric FRET (RO, BAY, DEM) significantly, as did known $\alpha \mathrm{SN}$ modulator EGCG. Experiments were performed with $n=3 ;{ }^{*} p<0.05,{ }^{* *} p<0.01,{ }^{* * *} p<0.001$, ns indicates no significant difference as determined by Student's $t$ test.

accumulated over $0.5 \mathrm{~s}$ ). It is also possible that the effect in $\mathrm{SH}$ SY5Y cells is more pronounced due to interactions of the compounds with other protein machinery involved in aSNinduced cell death. Indeed, DEM is a known calpain inhibitor ${ }^{74}$ and can play an active role in ameliorating calpain-dependent NLRP3 inflammasome activation, a known effector of aSN-induced pathology ${ }^{75-80}$. The interplay between potential MOA, whether direct binding of compounds to aSN or indirect MOA (e.g., via inhibition of calpain), are discussed at length below.

We investigated one potential MOA for compounds $\mathrm{RO}$ and DEM in our SH-SY5Y cytotoxicity model. These compounds might rescue aSN-induced cytotoxicity by modulating aSN expression $^{81,82}$. SH-SY5Y cells transfected with empty-vector or unlabeled WT aSN were treated with DMSO, RO, or DEM at $1 \mu \mathrm{M}$ concentration. Supplementary Fig. 15 shows that the drug treatment interestingly increases total aSN expression levels. This effect may be due to prolonged survival of cells expressing higher levels of aSN, which would be lost in DMSO-treated samples or as a result of shifting monomer:oligomer ratios $^{82}$. Studies have shown that there is a fine balance in aSN expression, where too much can lead to aggregation, but aSN expression can also be neuroprotective ${ }^{81,83-86}$.

Secondary assay: primary neuron model of aSN PFF pathology Further analysis of hits RO, BAY, and DEM in an aSN PFF model also exhibited some protection against later-stage aSN pathology. As described previously, numerous in vivo and in vitro alphasynucleinopathy mouse models have been developed using treatment with sonicated aSN PFF to model pathology. PFF treatment of primary mouse hippocampal neurons results in neurotoxicity and increased phosphoserine-129 $\left(\mathrm{ps}^{129}\right)$ immunoreactivity, a marker for alpha-synucleinopathy pathology ${ }^{87,88}$. These effects can be produced using either human-PFF or murine-PFF with the murine-PFF providing a stronger response. Primary neurons (7 days in vitro (DIV)) were treated for 12 days with murine-PFF or PBS and $1 \mu \mathrm{M}$ hit compound or DMSO (compound vehicle). Neurons were fixed and stained for NeuN (neuronal nuclei), Map2 (neurite and neuronal morphology), and 81a $\left(\mathrm{ps}^{129}\right.$ ) (see Supplementary Fig. 16). PFF treatment results in a significant loss of neurons (NeuN count, Supplementary Fig. 17) and a significant increase in pathology staining (e.g., normalized 81a/ NeuN) that is unchanged with DMSO treatment (Fig. 8b). Treatment with $1 \mu \mathrm{M}$ of either BAY or DEM reduces the $\mathrm{pS}^{129}$ phenotype; however, treatment with RO had little to no effect. 


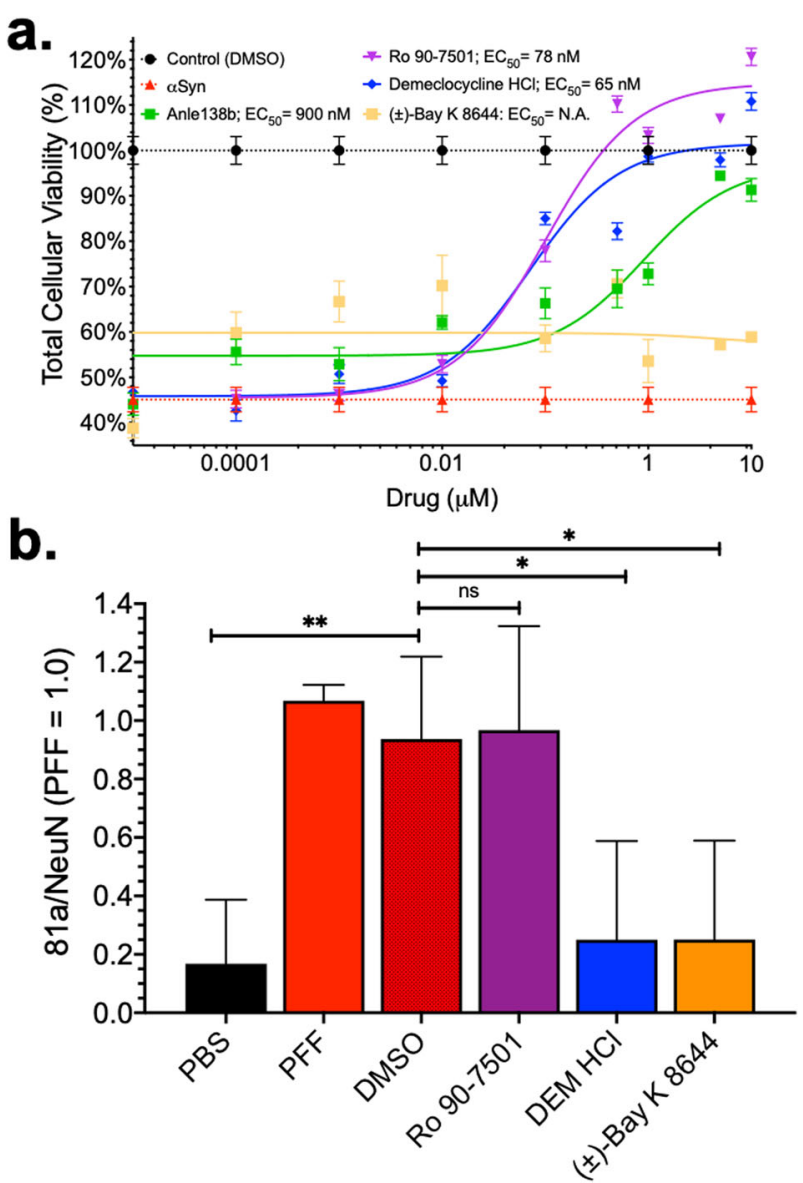

Fig. 8 Cytotoxicity in SH-SY5Y cells and primary neuron pathology model demonstrate hit compound rescue of aSN induce pathology. a Overexpression of $\alpha \mathrm{SN}$ in SH-SY5Y cells results in reduced cellular viability ( $44 \%$, red dashed line) as determined by CytoTox-Glo cytotoxicity assay. Treatment with control compound Anle138b as well as hit compounds RO and DEM demonstrate a dose-dependent rescue of toxicity with an $\mathrm{EC}_{50}$ of 900,78 , and $65 \mathrm{nM}$ for Anle138b, RO, and DEM, respectively. Biosensor hit BAY did not rescue the SH-SY5Y cell toxicity. $\mathbf{b}$ Using an $\alpha$ SN PFF-induced pathology model in primary mouse neurons, we evaluated the capacity of our hit compounds to rescue $\alpha$ SN PFF seed-associated pathology (phospho- $S^{129}$ staining). PFFs were treated at $\sim 415 \mathrm{ng}$. Two of our hit compounds reduced the $\alpha \mathrm{SN}$ pathology (BAY and DEM). Representative micrographs of each system are presented in Supplementary Fig. 14 with quantification of cytotoxicity via NeuN counting in Supplementary Fig. 15. $N=3$ independent experiment; ${ }^{*} p<0.05,{ }^{* *} p<0.01$, ns indicates not significant.

Reduction of $\mathrm{pS}^{129}$ signal in our co-treatment model (e.g., where both PFF and compound are administered simultaneously) could suggest either a rescue of PFF-induced pathology or inhibition of the $\mathrm{pS}^{129}$ signal through reduction of endogenous aSN phosphorylation. Future studies focused on hit-to-lead optimization will focus on these nuances to resolve hit compound's MOA.

\section{Secondary assay: differentiated SH-SY5Y cell model of aSN PFF pathology}

We next explored an orthogonal PFF pathology model that monitors $\mathrm{pS}^{129}$ signal in differentiated SH-SY5Y cells treated with sonicated human aSN PFF and show significant effects for each compound (Supplementary Fig. 18). We observe a distribution of $\mathrm{pS}^{129}$ immunoreactive bands that we delineate as monomeric, oligomeric, and high-molecular-weight (HMW) aSN species as well as a series of non-specific bands that are present across all samples (Supplementary Fig. 18a, b). Quantifying total $\mathrm{pS}^{129}$ (excluding non-specific bands) shows a significant decrease in total $\mathrm{pS}^{129}$ signal for $\mathrm{RO}$, a significant increase in total $\mathrm{pS}^{129}$ for $B A Y$, and a non-significant change for DEM (Supplementary Fig. 18c). Obvious differences between these data and those from the primary mouse neurons are noted here and discussed below. Parsing the data more closely, Supplementary Fig. 18d shows that all three compounds strongly alter the relative ratios of the various $\mathrm{pS}^{129}$ assemblies. In particular, all three hits drove a reduction in monomeric $\mathrm{pS}^{129}$ and an increase in $\mathrm{HMW} \mathrm{pS}^{129}$. Interestingly, two of the three hits (RO, DEM) and a control compound (Baicalin) also significantly reduced the low-molecular-weight (LMW) oligomer fraction.

\section{Secondary assay: interactions with aSN monomers}

Results from our intra-protomeric aSN biosensor FRET response suggested that hit compounds RO, Bay, and DEM may be modulating aSN oligomers by changing their conformation. The intra-protomeric biosensor is unable to distinguish between changes in monomer or oligomer conformation. To investigate the effects of our hit compounds on monomer conformation, we used smFRET and PrOF NMR. These biophysical techniques are performed under conditions where aSN is predominantly monomeric.

SmFRET experiments were performed using two different labeled aSN constructs (residues 9-72 and residues 54-130) that report on intra-monomeric FRET (e.g., monomer conformation) of the N-terminal and C-terminal domains of aSN. Neither Bay nor DEM showed a significant change in smFRET histograms (Supplementary Fig. 19 and Supplementary Table 2) indicating that these compounds do not modulate monomeric conformation. We were not able to evaluate RO with smFRET, due to interactions of RO with the AlexaFluor-594 label.

PrOF NMR provides a perturbation-free method to monitor aSN conformation and structure through the incorporation of F19labeled 3-fluoro-Tyrosine into recombinant protein ${ }^{89}$. The resting PrOF spectra for F19-labeled aSN shows three resonances that have been previously assigned to the four tyrosine residues (Supplementary Fig. 20a) ${ }^{89}$. Titration of hit compounds RO and DEM with fluorinated aSN shows no significant chemical shifts or resonance broadening (Supplementary Fig. 20b, c) except for high concentrations of RO (in excess of 5:1 compound-to-protein ratio). These results support a lack of binding to $\operatorname{aSN}(\mathrm{m})$. Results from both smFRET and PrOF NMR suggest that the potential MOA for these hit compounds is likely not via direct binding or interaction with $\operatorname{aSN}(\mathrm{m})$.

\section{Secondary assay: modulation of aSN oligomers}

Next we explored our hit compounds effect on recombinant aSN oligomers via a modified aSN oligomer assay adapted from Otzen et al. $^{90}$. Purified recombinant, $\operatorname{aSN}(\mathrm{m})(350 \mu \mathrm{M}, 5 \mathrm{mg} / \mathrm{mL})$ was incubated with each compound $(70 \mu \mathrm{M}$; a 1:5 compound:protein ratio) for $12 \mathrm{~h}$ under continuous shaking (900 RPM, $37^{\circ} \mathrm{C}$ ). After oligomerization, an aliquot of each sample was cross-linked in $1.6 \%$ paraformaldehyde (PFA) and characterized via SDS-polyacrylamide gel electrophoresis (PAGE) western blot analysis $^{91}$. Figure $9 a$ shows the resulting products from our oligomerization assay for non-cross-linked and cross-linked samples. Using the cross-linking, we are able to investigate SDS soluble assemblies that would be otherwise dissociated. There are clear changes in the distribution of bands with different compound treatments. Experimental replicates were processed on the same blot (Supplementary Fig. 21), with densitometry quantification based on monomer, LMW oligomers, and HMW oligomers (Fig. 9b). Each species was normalized to the DMSOtreated sample. There was a significant decrease in monomer and 
a.

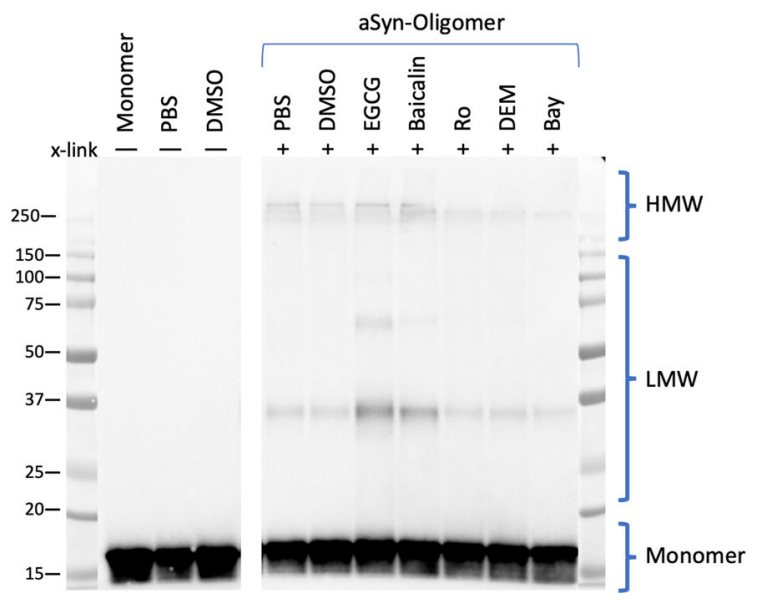

b.

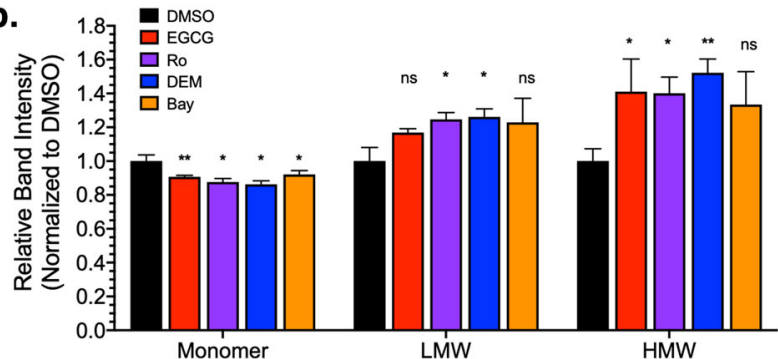

Fig. 9 Recombinant aSN oligomerization assay. a Cross-linked aliquots from recombinant $\alpha S N$ oligomerization assay were ran via SDS-PAGE, transferred, and probed for total $\alpha$ SN with antibody Syn 1 . Samples before and after cross-linking were evaluated and densitometry was performed by classifying $\alpha \mathrm{SN}$ assemblies as monomer, low-molecular-weight (LMW) oligomers, or highmolecular-weight (HMW) oligomers. b Densitometry analysis on cross-linked $\alpha S N$ oligomers (see Supplementary Fig. 18) illustrate a significant change in monomer (decrease) and both oligomers (increase) with compound treatment. All compounds were at substoichiometric ratio (1:5, compound: monomer). $N=3$ independent experiments; ${ }^{*} p<0.05,{ }^{* *} p<0.01$, ns indicates no significant difference as determined by Student's $t$ test.

subsequent increase in LMW and HMW oligomers for almost all hit compound conditions.

To explore the presence of $\beta$-sheet content in these oligomers, an aliquot was removed prior to cross-linking, diluted into $100 \mu \mathrm{M}$ ThT working solution, and ThT fluorescence was monitored to detect the presence of $\beta$-sheet. Supplementary Fig. 22 presents each sample ThT content normalized to the PFF signal. We observed an increased ThT content in the aSN oligomers (aS-O) that was not affected by DMSO (vehicle) treatment. Both control compounds, EGCG and Baicalin, are known modulators of aSN aggregation and demonstrate a complete loss of ThT signal. The three hit compounds tested had either no effect (RO) or increased the ThT signal (DEM and Bay) over DMSO, albeit not to the same extent as PFFs.

These effects on aSN oligomers suggest that our hit compounds interact with aSN oligomers, increasing the overall oligomer fraction and modulate the corresponding aSN oligomer structure, supporting the finding from our inter- and intra-protomeric FRET biosensors.

\section{Secondary assay: inhibition of aSN fibrillization (non-seeded and seeded aggregation)}

ThT aggregation assays monitor the kinetics and extent of fibril formation due to spontaneous and seeded aggregation ${ }^{26,92-95}$.
The cascade of spontaneous aSN fibrillization includes formation and maturation of oligomeric aSN assemblies ${ }^{96,97}$. Figure 10 presents spontaneous ThT aggregation for $\operatorname{aSN}(\mathrm{m})(140 \mu \mathrm{M})$ with or without hit compounds RO, DEM, and Bay $(70 \mu \mathrm{M}$; a substoichiometric ratio, 1:2, compound:protein). Here we observe significant ThT signal reduction for RO with lesser degrees of attenuation by Bay and DEM, respectively.

Figure 11 and Supplementary Fig. 23 present results from a dose-response-seeded aggregation assay. Vehicle, control, and hit compounds were incubated with $15 \mu \mathrm{M}$ aSN $(\mathrm{m})$, doped with $5 \%$ freshly sonicated aSN PFF in PBS under mild agitation (Supplementary Fig. 23a). Seeded monomer samples underwent rapid elongation, whereas monomer-only samples did not spontaneous aggregate during the span of the experiment (Supplementary Fig. 23b). Titration of control and hit compounds were run in triplicate; representative traces of hit compounds are illustrated in Fig. 11a with a full summary of each titration detailed in Supplementary Fig. 23c-h. $I C_{50}$ was determined for each compound by determining the max ThT intensity for each concentration (Fig. $11 \mathrm{~b})$. Both RO and DEM demonstrated similar IC 50 as control EGCG (931 nM, $1.0 \mu \mathrm{M}$, compared to $1.1 \mu \mathrm{M}$ for EGCG).

\section{DISCUSSION}

The folding/misfolding pathways that connect aSN monomers, oligomers, and fibrils (Fig. 12) are highly stochastic, and the degree of heterogeneity of molecular states may never be fully grasped. Even fibril structures are turning out to be variable, with distinct inter-monomeric motifs emerging under differing conditions and pathologies ${ }^{98-102}$. But the heterogeneity is of a different order, and is most confounding, for oligomers: do they have any discernable, stable structural motifs? Do these motifs contribute to the seeding competency of aSN assemblies? And, if so, are any of those motifs structured enough to constitute robust and reproducible small-molecule-binding sites? These uncertainties do not mean that small molecules targeting oligomers cannot act as high-potency inhibitors, as we have shown here that they can. Instead, the heterogeneous and metastable nature of aSN oligomers may force a re-thinking of conventional approaches to drug discovery. For example, rather than optimizing lead compounds to selectively and potently block an active site, we may need a cocktail of small molecules that act in a statistical way, collectively shifting the ensemble of accessible structural states of aSN to bias the folding pathway, shifting oligomers from toxic to non-toxic assemblies (Fig. 12).

A fundamental question arises from this framing: what is the ideal MOA for a small molecule, or a set of small molecules, that target aSN oligomers? One possibility is that the most efficient small molecules should directly bind aSN, in any of its myriad states, and perturb local structural elements-e.g., disrupts motifs or inhibit seeding - to prevent or reduce the population of toxic oligomers or spread of aSN pathology. This perspective is supported by a recent HTS campaign targeting SDS-induced aSN aggregation, where the most potent compounds predominantly targeted and bound to aSN $(m)$, inhibiting subsequent aggregation $^{37}$. We started from this traditional vantage point, assuming that our best hits would increase FLT (decreased FRET) through interacting with aSN $(\mathrm{m})$. We based this on our experience with a similar screening platform developed to target tau oligomers, where our most functionally efficient hit decreased $\mathrm{FRET}^{47}$. To our surprise, the compounds with the most potent functional profiles (DEM and RO) increased FRET in our interprotomeric biosensor, while the one hit that decreased FRET (SB206553) had no effect on cytotoxicity. It is possible that no small molecules in the LOPAC library, or any single compound for that matter, can fully dissociate or prevent oligomers. More likely, these small molecules either increase the extent of oligomerization or alter the organization of the protomers within the 
oligomers in such a way that (1) brings the fluorophores in closer proximity (possibly reflecting increased compaction) and (2) concomitantly buries or exposes motifs within the oligomer that both slow fibrillization (Figs. 10 and 11 and Supplementary Fig. 23) and reduce toxicity (Fig. 8 and Supplementary Figs. 14 and 17) in as yet unknown ways.

Determining whether these motifs exist, and what they are, may yet be possible. High-resolution structural measurements in cells are difficult, but progress is being made. For example, recent NMR and electron paramagnetic resonance experiments in cells have shown that aSN maintains disorder in the cellular milieu ${ }^{103}$. And deep mutational sequencing techniques may be useful in mapping a binding site ${ }^{104}$. The lifetime FRET measurements made here capture lower-resolution information but nonetheless are an extremely sensitive cellular method that allows us to rapidly identify small molecules that cause minute structural changes in

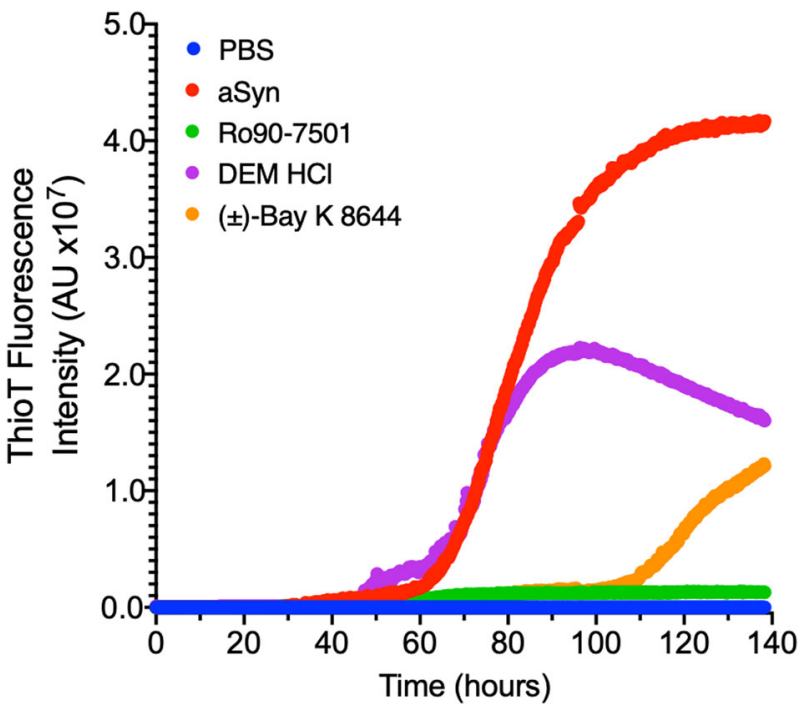

Fig. 10 Monomer induced aggregation of aSN (non-seeded Thioflavin-T assay). Recombinant $\alpha \mathrm{SN}$ was incubated at $140 \mu \mathrm{M}$ monomer $(70 \mu \mathrm{M}$ compound) under continuous shaking (900 RPM) at $37^{\circ} \mathrm{C}$. All three hit compounds modulated $\alpha \mathrm{SN}$ fibrillization with RO having a more significant effect over Bay and DEM, respectively.
aSN oligomers. This sensitivity is enhanced with our use of multiple, distinct FRET biosensors. Coupling the FRET response from the inter- and intra-protomeric biosensors provide further insight into each Hit's MOA. The collective results of our FRET, cytotoxicity, $\mathrm{pS}^{129}$ pathology, smFRET and PrOF NMR, and in vitro oligomerization assay suggest that our hit compounds bind to and induce an increase in aSN oligomerization, biased toward nontoxic aSN oligomers where constituent monomers are in more extended conformations. That both DEM and RO are effective at low $\mathrm{nM}$ concentrations in cells (Fig. 8a), increase the formation of aSN oligomers (Fig. 9 and Supplementary Figs. 21 and 22) and slow or prevent growth of pre-formed fibrils in vitro (Figs. 10 and 11 and Supplementary Fig. 23), and reduce the presence of $\mathrm{pS}^{129}$ positive LMW oligomers in cells (Supplementary Fig. 18) may be some of the first evidence to suggest a targetable binding site that persists from oligomer to fibril.

Though we are far from providing definitive proof of persistent, robust targetable motifs, our $\mathrm{pS}^{129}$ data from both the mouse primary neuron model and SH-SY5Y model are also at least consistent with this possibility. Given the vastly different experimental platform of these experiments compared to our screening platform (e.g., mouse vs. human aSN in the primary neuron assay, spontaneous oligomerization vs. exogenous PFF treatment), that we saw an effect across systems is compelling. One possibility is that the compounds are directly binding to the PFF or oligomers in the PFF mixture, altering their growth and stability, or sterically altering accessibility to modifying enzymes. This would imply the existence of a persistent and robust motif targeting human aSN oligomers in HEK293 cells, SH-SY5Y cells (RO and DEM but not BAY for cytotoxicity), mouse aSN PFFs in primary neurons (DEM and BAY), and human aSN PFFs in differentiated SH-SY5Y cells (RO and DEM, and not BAY, reduce oligomers; RO, DEM, and BAY increase HMW assemblies).

In a recent study, the Volpicelli-Daley group detailed the nuanced in vivo pathological phenotypes associated with aSN oligomers and fibrils, demonstrating that, although both nonseeding-competent oligomers and PFF are capable of inducing neurotoxicity and synaptic deficits, only the latter PFF treatment resulted in PD-like staging of pathology and motor/behavioral phenotypes $^{105}$. Although our cellular FRET biosensors monitor changes in oligomeric aSN, our hit compounds do display the capacity of disrupting both PFF-induced pathological phenotypes (Fig. 8 and Supplementary Figs. 16-18) and seeded aSN
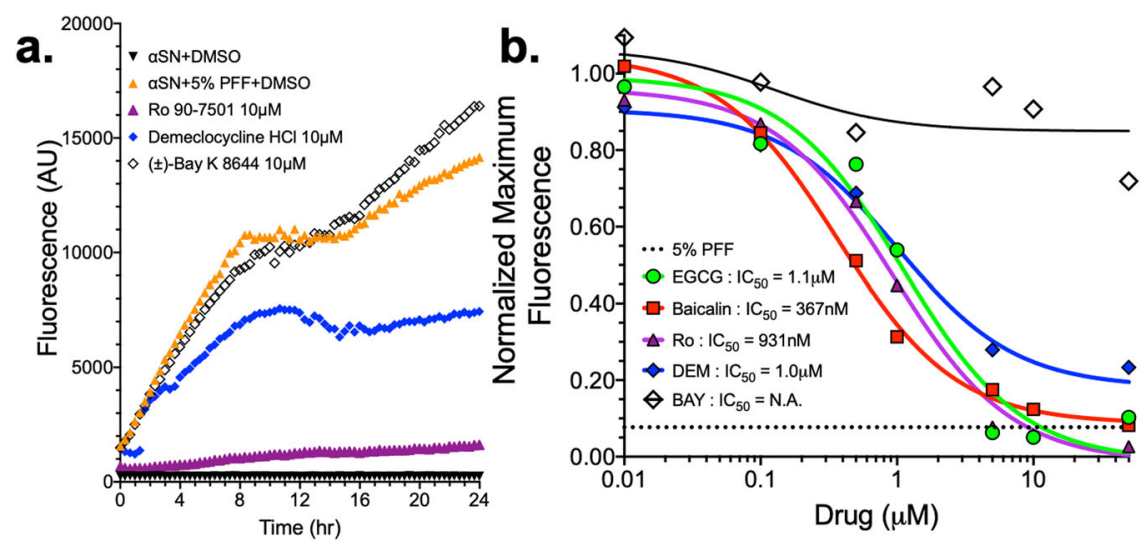

Fig. 11 Seeded Thioflavin-T aggregation assay shows direct protein interaction for subset of hit compounds. Seeded ThT aggregation assay was performed using $15 \mu \mathrm{M}$ monomeric $\alpha \mathrm{SN}$ doped with $5 \%(0.75 \mu \mathrm{M}$ sonicated $\alpha \mathrm{SN}$ pre-formed fibril (PFF) seeds) in a 384 -well plate under slow agitation (300 RPM) at $37^{\circ} \mathrm{C}$. a Control samples for the aggregation include PBS (black), $\alpha \mathrm{SN}$ monomer (brown), 5\% $\alpha \mathrm{SN}$ PFF (red), and the $15 \mu \mathrm{M}$ monomer $+5 \%$ PFF mixture (orange). Dose-response curves were made with a titration of compounds. Some hit compounds had little to no effect on aggregation, even at $50 \mu \mathrm{M}$ (e.g., SB206553). In contrast, RO and DEM both show potent effects relative to Baicalin and EGCG controls. BAY does have some response, but it lags behind other hit compounds. b Normalizing the initial peak in ThT data allows us to estimate an $\mathrm{IC}_{50}$ for each hit. Control compounds EGCG and Baicalin show $1.1 \mu \mathrm{M}$ and $387 \mathrm{nM}$ potency, whereas RO and DEM have IC50 of $931 \mathrm{nM}$ and $1.0 \mu \mathrm{M}$. Although there is a small trend with BAY, the data cannot be fit to resolve an $\mathrm{IC}_{50}$. 


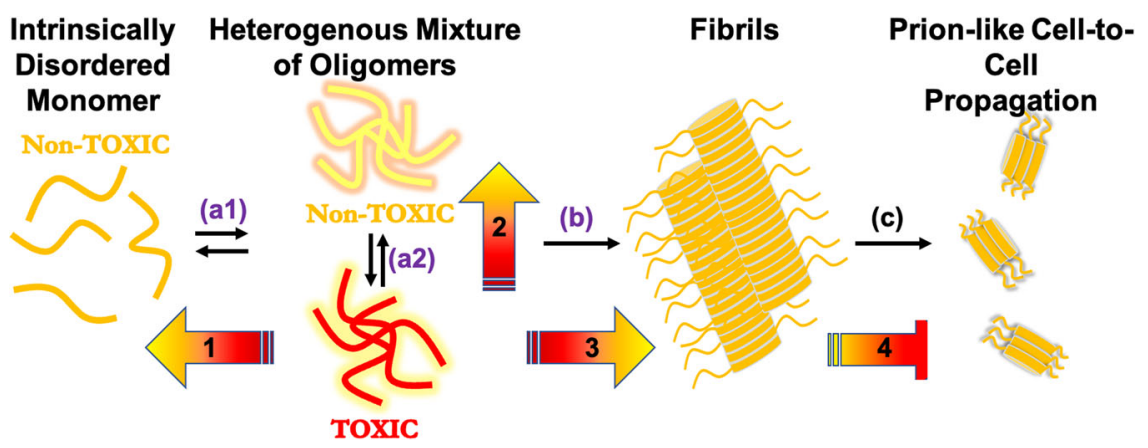

Fig. 12 Monitoring spontaneous aSN oligomers and conformation using live cell fluorescence lifetime readouts. The intrinsically disordered $\alpha \mathrm{SN}$ monomer is capable of misfolding into oligomers and fibrils, producing toxic assemblies that have been implicated in the pathology of disease. Oligomers comprise a heterogeneous mixture of metastable assemblies that have proven difficult to monitor with high precision and accuracy, so most current strategies target the irreversible formation of large assemblies (fibrils, arrow b). However, disruption of fibrils can induce toxicity due to elevated levels of toxic oligomers. Our cellular FLT-FRET biosensors monitor reversible spontaneous oligomerization (a1) in addition to structural remodeling within the oligomer assembly (e.g., conversion between toxic and non-toxic oligomers, a2), while also monitoring downstream processes such as fibrillization (b) and seeded aggregation (c) with high sensitivity. This study demonstrates the capacity of our drug discovery pipeline to identify compounds that modulate the toxicity of these $\alpha \mathrm{SN}$ assemblies (gradient arrows 1-4), thereby shifting the equilibrium of these heterogeneous assemblies toward non-toxic species.

fibrillization (Fig. 11 and Supplementary Fig. 23). These results suggest that these hit compounds may be able to disrupt aSN PFF seeding and cell-to-cell spread, highlighting an exciting direction for future studies to evaluate our hits in more complex and physiologically relevant neuronal or animal models. Nevertheless, the ability of our drug discovery platform to identify hit compounds that can target a multitude of therapeutic MOAs further demonstrates the broad potential application of our cellular aSN oligomer FRET biosensors.

A second, indirect MOA is possible as well and may explain why BAY showed an effect in the HEK293 cells and the mouse model but not in SH-SY5Y cells. Small molecules that alter a wide range of auxiliary cellular processes (e.g., activating the unfolded protein response, the endoplasmic reticulum stress response, etc.) could indirectly alter oligomeric structures (HEK293 cells), reduce cell death (SH-SY5Y), reduce phosphorylation of Ser129 (mouse primary neurons), and alter the distribution of $\mathrm{pS}^{129}$ assemblies (differentiated SH-SY5Y cells) without ever binding to aSN. But, again, the inherent difficulty in assessing direct binding of small molecules to a heterogeneous array of structured and unstructured oligomers in cells makes a definitive assignment of MOA, direct or indirect, challenging. Despite this, it is possible that compounds that act indirectly may ultimately prove the most potent, as they do not face the hurdle of needing to alter a complex heterogeneous ensemble of oligomers.

The collection of experiments presented here do not definitively elucidate the hit compounds' MOA for altering aSN oligomers, cytotoxicity, and pathology. Indeed, in our two distinct $\mathrm{pS}^{129}$ pathology models we observe model-dependent differences: BAY and DEM reduce total $\mathrm{pS}^{129}$ in primary mouse neurons (Fig. 8) and only $\mathrm{RO}$ reduces total $\mathrm{pS}^{129}$ in differentiated SH-SY5Y cells (Supplementary Fig. 18). These inconsistencies undoubtedly result from the multitude of differences between the two assays (mouse vs. human aSN PFF; primary mouse neuron vs. human neuronal cell line; treatment duration (12 days vs. 2 days) or detection methodology (immunofluorescence cytochemistry vs. immunoblots). Hit compound effects become even more intriguing with the significant reduction in monomer $\mathrm{pS}^{129}$ fraction (RO, BAY, and $\mathrm{DEM}$ ) and $\mathrm{LMW}$ oligomer $\mathrm{pS}^{129}$ fraction (RO and DEM) with a relative increase in the fraction of HMW assemblies (Supplementary Fig. 18). These apparent increases in HMW species could be due to a number of things (e.g., changes in PFF cellular uptake; changes in PFF clearance; remodeling of PFF or $\mathrm{aSN}(\mathrm{m})$ ). Furthermore, it is likely that these HMW assemblies are a complex mixture of toxic and non-toxic species whose acute properties can be difficult to delineate, especially in cellular experiments. The effect of Baicalin (control compound in Supplementary Fig. 18) in our SH-SY5Y pS $^{129}$ assay highlights this challenge, as Baicalin is known to remodel aSN PFF into non-toxic amorphous aggregates, yet we still observe a strong $\mathrm{HMW} \mathrm{pS}^{129}$ signal. RO has been previously shown to inhibit $A \beta$ fibrillization through interaction with structural intermediates ${ }^{106}$ and may have a similar direct MOA with aSN. DEM (a tetracycline derivative) may share properties with other tetracycline-like molecules (i.e., doxycycline), which has been shown to induce off-pathway oligomerization of aSN ${ }^{107}$

Nevertheless, the primary focus of this study is the demonstration of our HTS platform's ability to identify small molecules capable of modulating aSN oligomer structure and associated pathological phenotypes. Our screening platform, predicated on FRET measurements that monitor alterations in aSN's oligomeric state, has successfully identified compounds that shift the relative ratios of the various oligomeric populations (Figs. 3 and 9 and Supplementary Figs. 18, 21, and 22). The exact mechanism by which those alterations change aSN pathology, a subject of essential debate in the field, should be the subject of future work.

Cellular aSN FRET biosensors have been previously developed and used successfully as sensitive biomarkers to detect seedingcompetent aSN assemblies in patient-derived biofluids ${ }^{108}$. These earlier biosensors have been focused on characterizing cell-to-cell seeding and fibrillar aSN. The biosensor systems described here can be similarly used and the FLT detection scheme may improve the overall sensitivity of these approaches. Comparisons between our biosensor system and those previously developed are planned for future studies. BiFC has also been used before as a means to monitor aSN oligomerization ${ }^{42,44}$. Direct protein-protein interaction and fluorophore maturation is required for BiFC fluorescence. However, a disadvantage of using these systems for smallmolecule screening is that it requires the compounds to dissociate the aSN assemblies by disrupting both the BiFC tag and target protein-protein interaction. In each of these approaches, including ours, there is the concern regarding XFP-induced artifacts. To reduce potential false positive hit detection, XFP fusion proteins are used solely in our primary HTS and confirmatory counter screens and FRET dose-response experiments. All other cellular assays are performed with unlabeled WT aSN (no fusion constructs). Furthermore, for assays with XFP fusion constructs all experiments are repeated with control biosensors to rule out non-specific FRET contributions. 
To conclude, our inter- and intra-protomeric FRET aSN biosensors provide a new platform for evaluating aSN conformation and oligomerization. Extensions of our screening platform moving forward will focus on finding increasingly potent and central nervous system-penetrant small molecules and will be designed to answer many open questions. For example, screening in a variety of neuronal cell lines can address the ongoing question of whether variations in cellular milieu alters oligomeric structures (Are there common or disparate hits?), and screening against known familial mutants could clarify whether those also associate with differences in the structures of these toxic assemblies.

\section{METHODS}

\section{Molecular biology}

To generate the aSN FRET constructs, aSN CDNA was cloned into an EGFPlinker-TagRFP plasmid (linker contains 32 amino acids, GFP-32AA-RFP) that was previously characterized ${ }^{50}$. QuikChange mutagenesis (Agilent Technologies, Santa Clara, CA) was performed to monomerize the GFP via A206K mutation and to produce the donor GFP-aSN construct ${ }^{109}$. Subsequent QuikChange mutagenesis was used to remove the 32 amino acid linker and stop codon after aSN (creating GFP-aSN-RFP construct) as well as to excise the GFP (creating unlabeled aSN and aSN-RFP constructs). The aSN A53T mutation was introduced into all four plasmids. Primers for all QuikChange mutagenesis are detailed in Supplementary Table 3. All biosensor plasmid constructs were sequenced for confirmation (ACGT, Wheeling, IL).

\section{Cell culture}

HEK293 and SH-SY5Y cells (ATCC) were cultured in phenol red-free Dulbecco's Modified Eagle Medium (Gibco) supplemented with $2 \mathrm{mM} \mathrm{L-}$ Glutamine (Invitrogen), heat-inactivated 10\% fetal bovine serum (Gibco), $100 \mathrm{U} / \mathrm{mL}$ penicillin, and $100 \mu \mathrm{g} / \mathrm{mL}$ streptomycin (Gibco). Cell cultures were maintained in an incubator with $5 \% \mathrm{CO}_{2}$ (Forma Series II Water Jacket $\mathrm{CO}_{2}$ Incubator, Thermo Scientific) at $37^{\circ} \mathrm{C}$. The inter-protomeric (oligomer) and intra-protomeric (oligomer and conformation) aSN FRET biosensors were generated by transiently transfecting HEK293 cells using Lipofectamine 3000 (Invitrogen) with GFP-aSN and aSN-RFP (1:8 DNA plasmid concentration ratio) or GFP-aSN-RFP plasmid, respectively. The effectiveness of HEK293 cells transfected with FRET constructs as an HTS platform has been demonstrated in our previous work ${ }^{46-52,66}$.

\section{LOPAC library and liquid handling}

The LOPAC (Sigma-Aldrich) contains 1280 compounds that span marketed drugs, failed development candidates, and naturally occurring compounds that have well-characterized activities with widely described biological effects. The library is originally formatted in 96-well mother plates and dispensed across four 384-well and/or one 1536-well flat, black-bottom polypropylene plates at $10 \mu \mathrm{M}$ final concentration/well $(50 \mu \mathrm{L}$ final volume for 384-well; $10 \mu \mathrm{L}$ for 1536 wells) using an automated Echo acoustic liquid dispenser from Labcyte (Sunnyvale, CA, USA). DMSO (matching \%v/v) was loaded as in-plate no-compound negative controls to make a total of 960 wells (384-well plates) or 256 wells (1536-well plate). The plates were sealed and stored at $-20^{\circ} \mathrm{C}$ until use.

Two days prior to screening, HEK293 cells were transfected using Lipofectamine 3000 with GFP-aSN/aSN-RFP (aSN oligomer FRET biosensor) or GFP-aSN-RFP (aSN monomer conformation FRET biosensor) in 15x $100 \mathrm{~mm}$ plates $\left(6 \times 10^{6}\right.$ cells/plate). On each day of screening, the compound plates were equilibrated to room temperature $\left(\mathrm{RT} ; 25^{\circ} \mathrm{C}\right)$. The cells were harvested from the $100-\mathrm{mm}$ plates by incubating with TrypLE (Invitrogen) for $2 \mathrm{~min}$, washed three times in PBS by centrifugation at $200 \times g$, and filtered using 70- $\mu \mathrm{m}$ cell strainers (BD Falcon). Cell viability prior to screen was assessed using a trypan blue assay and confirmed to be $>80 \%$. Cells were diluted to $1.5 \mathrm{E} 6$ cells $/ \mathrm{mL}$. Expression of GFP-aSN and the aSN biosensors were confirmed by fluorescence microscopy prior to each screen (EvosFL-Auto microscope, Thermo Fisher Scientific, USA). During screening, cells $(50 \mu \mathrm{L} /$ well for 384-well; $10 \mu \mathrm{L} /$ well for 1536-well) were dispensed by a Multidrop Combi liquid dispenser (Thermo, Pittsburg, PA, USA) into the assay plates containing the compounds and allowed to incubate at RT for $90 \mathrm{~min}$ before recording with the FLT-PR or SUPR (both instruments manufactured by Fluorescence Innovations Inc., both owned and provided by Photonic Pharma LLC), as described previously ${ }^{46}$. Reproducible FRET hits for retesting were purchased from Tocris (Minneapolis, MN, USA), Sigma (St. Louis, MO, USA), or Invitrogen (Carlsbad, (A, USA), depending on availability.

\section{HTS and FLT data analysis}

As described previously ${ }^{46,47,50}$, time-resolved fluorescence waveforms for each well were fit with single-exponential decays using the least-squares minimization global analysis software to give donor-acceptor lifetime $\left(\tau_{\mathrm{DA}}\right)$ and donor-only lifetime $\left(\tau_{\mathrm{D}}\right)$. FRET efficiency $(E)$ was then calculated based on Eq. 1.

$E=1-\left(\frac{\tau_{\mathrm{DA}}}{\tau_{\mathrm{D}}}\right)$

Assay quality was determined using control EGCG and hit compound DEM as positive controls and DMSO as a negative control and calculated based on Eq. $2^{110}$,

$Z^{\prime}=1-\frac{3\left(\sigma_{\mathrm{p}}+\sigma_{\mathrm{n}}\right)}{\left|\mu_{\mathrm{p}}-\mu_{\mathrm{n}}\right|}$

where $\sigma_{\mathrm{p}}$ and $\sigma_{\mathrm{n}}$ are the SDs of the observed $\tau_{\mathrm{DA}}$ values and $\mu_{\mathrm{p}}$ and $\mu_{\mathrm{n}}$ are the mean $\tau_{\mathrm{DA}}$ values of the positive and negative controls.

Interfering compounds were flagged as potential false positives by a set of stringent FC filters based on spectral analysis of each well from the LOPAC screen ${ }^{45,46}$. After removal of FCs, a histogram of the FLT distribution from all compounds in the screen was plotted and fit to a Gaussian curve to obtain the mean $(\mu)$ and SD $(\sigma)$. A hit was defined as a compound that attenuated the FLT efficiency by $>5 \sigma$ relative to the mean $\mu$ (Supplementary Fig. 12).

Initial biosensor characterization was performed via a titration of donor and acceptor DNA into HEK293 cells. Cells were transiently transfected and incubated for $48 \mathrm{~h}$, harvested, washed, and dispensed with 5 replicates for FLT characterization in the FLT-PR. Figure 1a illustrates a robust donor-only (1:0) FLT that undergoes increasing FLT attenuation with increased acceptor ratio through D:A of 1:12. There is a plateau in FLT reduction that correlates with overall donor signal to noise. Figure $1 \mathrm{~b}$ presents the corresponding FRET for each system determined via Eq. 1, where there is significant increase in basal FRET up to $D: A=1: 8$.

\section{FLT dose-response assay}

Compounds were tested in a FLT dose-response assay. The compound was dissolved to $10 \mathrm{mM}$ in DMSO, then serially diluted in 96-well mother plates. Each hit was re-screened for FLT dose response across eight concentrations $(1 \mathrm{nM}$ to $50 \mu \mathrm{M})$. Compounds $(0.5 \mu \mathrm{L})$ were transferred from mother plates into assay plates using a Mosquito HV liquid handler (TTP Labtech Ltd, UK). The preparations for aSN FRET biosensors were carried out as above.

\section{Spectral unmixing experiment and spectral data analysis}

For each biosensor system, the fluorescence emission spectrum was recorded and analyzed as described previously ${ }^{66}$. In brief, biosensor cells are harvested and prepared as for FLT-PR experiments and dispensed in either 1536- or 384-well plates (Greiner Bio-One) at a cell density of 1e6 cells $/ \mathrm{mL}$ ( 5 or $50 \mu \mathrm{L} /$ well, respectively). Each sample was excited using a 473-nm, continuous-wave laser and fluorescence emission spectra recorded across the entire visible spectrum with the 500-700-nm range used in spectral fitting.

The observed spectrum $F(\lambda)$ was fitted by least-squares minimization to a linear combination of component spectra

$F_{\text {fit }}(\lambda)=a F_{\mathrm{D}}(\lambda)+b F_{\mathrm{A}}(\lambda)+c F_{\mathrm{C}}(\lambda)+d F_{\mathrm{W}}(\lambda)$

where $D$ is donor, $A$ is acceptor, $C$ is cell autofluorescence background, and $\mathrm{W}$ is water Raman scattering and a-d are the coefficients determined from the fit to a series of basis functions defined for each component. The fitted spectrum is then determined using least-squares minimization with Matlab (MathWorks) ${ }^{66}$.

\section{Flow cytometry}

After biosensor cells were harvested and prepared for FLT-PR experiments, an aliquot of cells ( 1 1e6 cells in total) was collected and processed via flow cytometry. Biosensor-expressing cells were analyzed with a BD Accuri C6 
flow cytometer. Initial gating was defined with untransfected and single GFP- or RFP-transfected cells to establish baseline for the co-transfected biosensor cells. Cell analysis was complete after 2e5 cellular events were recorded.

\section{Cell cytotoxicity assay}

Cell cytotoxicity was measured using the CytoTox-Glo (Promega Corporation) Luminescence Assay Kit. SH-SY5Y human neuroblastoma cells were plated at a density of $5 \times 10^{6}$ cells/plate in $100 \mathrm{~mm}$ plate (Corning) and transfected with unlabeled WT aSN or equivalent vector-only control for $24 \mathrm{~h}$. The transfected cells were then plated at a density of 5000 cells/well in white solid 96-well plate (Corning) with a total volume of $100 \mu \mathrm{L}$, followed by treatment with hit compounds spanning a range of concentrations $(0.1 \mathrm{nM}$ to $100 \mu \mathrm{M})$, as well as DMSO-only controls, for another $48 \mathrm{~h}$. After incubation, $50 \mu \mathrm{L}$ of CytoTox-Glo Cytotoxicity Assay Reagent was added to all wells followed by mixing by orbital shaking and incubation in the dark for $15 \mathrm{~min}$ at RT. Luminescence readings were measured using a Cytation3 Cell Imaging Multi-Mode Reader luminometer (BioTek). After the first read, $50 \mu \mathrm{L}$ of Lysis Reagent was added, incubated for $15 \mathrm{~min}$ at RT, and a second read performed. Total viable cellular luminance was determined by the difference between the first and second luminescence signal and total cellular viability is determined by normalizing to the no-treatment samples.

\section{Primary hippocampal neuron cultures and fibril transduction}

Primary neuronal cultures were prepared from CD1 embryos on E16-18, as previously described ${ }^{58}$. Primary mouse neuron protocol was approved by the University of Pennsylvania Institutional Animal Care and Use Committee. Tissue culture plates and coverslips were coated with Poly-Dlysine (Sigma) before addition of cells. Neurons were plated in 96-well plates $\left(60,000\right.$ cells $\left./ \mathrm{cm}^{2}\right)$. Cultures were maintained in Neurobasal medium supplemented with B27 and Glutamax (all from Invitrogen).

PFF treatment was performed at 7 DIV and cultures were incubated for a further 12 days prior to fixation and analysis. Briefly, stock aSN PFFs were diluted in sterile DPBS (without $\mathrm{Ca}^{2+} / \mathrm{Mg}^{2+}$; Corning)-8 $\mu \mathrm{L}$ of aSyn + $392 \mu \mathrm{L}$ of DPBS into $1.5 \mathrm{~mL}$ Eppendorf tubes-and sonicated with a bath sonicator (Bioruptor Plus, Diagenode) for 10 cycles at high power (30 s on, $30 \mathrm{~s}$ off, at $10^{\circ} \mathrm{C}$ ). Sonicated PFFs were then further diluted to the indicated final concentration in neuronal media and added to cultures. PFF concentrations are expressed as the total equivalent aSN monomer content in the preparation. Cultures were treated with $200 \mathrm{nM}$ PFFs unless otherwise noted. Compounds were initially diluted in neuronal media before being added to the PFF neuronal media solution at a final concentration of $1-10 \mu \mathrm{M}$.

\section{PFF and compound treatment in differentiated SH-SY5Y cells}

SH-SY5Y cells were differentiated using reduced serum (3\%) media supplemented with $10 \mu \mathrm{M}$ retinoic acid for 3 days and then changed to serum-free media supplemented with $10 \mathrm{ng} / \mathrm{mL}$ brain-derived neurotrophic factor for the remainder of the experiment. Stock aSN PFFs were diluted and sonicated as described previously. SH-SY5Y cultures were then treated with $200 \mathrm{nM}$ PFF and PBS, DMSO, $1 \mu \mathrm{M}$ drug compound (RO, BAY, or DEM), or $50 \mu \mathrm{M}$ Baicalin. After $48 \mathrm{~h}, \mathrm{SH}-\mathrm{SY} 5 \mathrm{Y}$ were washed with PBS and harvested in cold lysis buffer with protease and phosphatase inhibitors, as previously described. Approximately $15 \mu \mathrm{g}$ of total protein was loaded onto a 4-20\% Criterion TGX midi gel and probed using Syn1 (BD Biosciences, \#610787; $1^{\circ} 1: 5000 ; 2^{\circ} 1: 10 \mathrm{~K}$ ) and $\mathrm{pS}^{129}$ aSyn (abcam, ab59264; $\left.1^{\circ} 1: 1000 ; 2^{\circ} 1: 5000\right)$ and a-tubulin (abcam, ab4074; $1^{\circ} 1: 1000 ; 2^{\circ} 1: 10 \mathrm{~K}$ )

\section{Immunocytochemistry and antibodies}

Cultured neurons were fixed by replacing media with warm PFA (4\% in PBS containing $4 \%$ sucrose) for 15 min at RT. Fixed neurons were washed three times with PBS and then blocked (3\% bovine serum albumin, 3\% fetal bovine serum in PBS) for $1 \mathrm{~h}$ at RT. Cells were then incubated in primary antibodies diluted in blocking buffer for $1 \mathrm{~h}$ at RT. Primary antibodies used in this study were: $\mathrm{p}-\mathrm{S} 129$ aSN (pSyn; CNDR mouse monoclonal $\lg _{2 a} 81 \mathrm{~A}_{\text {; }}$ 1:3000), NeuN (Millipore A60; 1:3000), MAP2 (CNDR rabbit monoclonal 17028: 1:3000). This was followed by washing with PBS three times and incubating for $1 \mathrm{~h}$ at RT in blocking buffer containing Alexa-Fluorconjugated isotype-specific secondary antibodies (Thermo Fisher). Cells were washed three times with PBS and 4,6-diamidino-2-phenylindole $(0.4 \mu \mathrm{g} / \mathrm{mL}$ in PBS) was added to visualize cell nuclei. Stained cells were imaged using an InCell 2200 (GE Healthcare Life Sciences). Images from 9 fields were collected from each well with a $\times 20$ objective. Image analysis and quantification was performed using the InCell Toolbox Analyzer software (GE Healthcare Life Sciences). The total integrated area for 81a immunopositivity was quantified for each sample and then normalized (divided) by the number of NeuN-positive objects in the fields examined. Our approach did not distinguish between ankyrin/puncta structures vs. more elongated pathologies that have also been described in this model system.

\section{Western blot analysis for biosensor expression}

To test the expression of aSN FRET biosensors, HEK293 cells were plated in a 6-well plate at a density of $1 \times 10^{6}$ cells/well and transfected with GFPaSN/aSN-RFP plasmids. Cells were lysed for $30 \mathrm{~min}$ on ice with radioimmunoprecipitation assay (RIPA) lysis buffer (Pierce RIPA buffer, Thermo Fisher Scientific) containing $1 \%$ protease inhibitor (Clontech, Mountain View, CA) and $1 \%$ phosphatase inhibitors (Millipore Sigma), and centrifuged at $15,000 \times g$ at $4{ }^{\circ} \mathrm{C}$ for $15 \mathrm{~min}$. The total protein concentration of lysates was determined by bicinchoninic acid (BCA) assay (Pierce), and equal amounts of total protein $(60 \mu \mathrm{g})$ were mixed with $4 \times$ Bio-Rad sample buffer and loaded onto 4-15\% Tris-glycine SDS-PAGE gels (Bio-Rad, Hercules, CA). Proteins were transferred to supported nitrocellulose membrane and probed using Syn 1 and $\mathrm{pS}^{129}$ aSyn antibodies against aSN. Blots were imaged on using a ChemiDoc MP imager and analyzed using Image Lab (Bio-Rad, Hercules, CA). All blots used for quantification derived from the same experiment and were processed in parallel.

\section{Triton-X 100 soluble/insoluble fractions}

HEK cells were transiently transfected with empty vector or GFP-aSN/aSNRFP as previously described. Cells were treated with either PBS or PFF seeds $(4 \mu \mathrm{g} / \mathrm{mL}) 24 \mathrm{~h}$ after transfection. After $24 \mathrm{~h}$ of treatment, cells were harvested in cold lysis buffer $(50 \mathrm{mM}$ Tris/ $\mathrm{HCl}$ pH 7.4, $150 \mathrm{mM} \mathrm{NaCl}, 5 \mathrm{mM}$ EDTA $\mathrm{pH}$ 8.0) with protease and phosphatase inhibitors and $1 \%$ Triton-X 100 and incubated on ice for $30 \mathrm{~min}$. The lysates were then centrifuged at $25,000 \times g$ for $60 \mathrm{~min}$ at $4^{\circ} \mathrm{C}$. The supernatant was collected as the Triton- $X$ 100 soluble fraction and the pellet was resuspended in lysis buffer with $2 \%$ SDS and sonicated for $10 \mathrm{~s}$ and collected as the Triton-X 100 insoluble fraction. Both fractions were boiled and then a BCA protein assay was used to quantify the protein concentration of the samples. Approximately $12 \mu \mathrm{g}$ of total protein was loaded onto a 4-20\% mini-Protean TGX gel (Bio-Rad, Hercules, CA), and blots were evaluated as described previously.

\section{Immunoprecipitation}

GFP-Trap magnetic agarose from Chromotek (Munich, Germany) were used for IP per the manufacturer's instructions. HEK cells were transiently transfected as previously described with aSyn biosensor plasmids and with appropriate controls [HEK control cells, GFP:aSN, GFP:aSN-RFP, GFP-aSN: RFP, GFP-aSN: aSN, GFP-aSN: aSN-RFP (D:A 1:8), and GFP-aSN: aSN-RFP (D: A 1:12)]. Cells were harvested in ice-cold native lysis buffer $48 \mathrm{~h}$ after transfection and agitated for $60 \mathrm{~min}$ at $4{ }^{\circ} \mathrm{C}$. The cellular lysates were centrifuged 12,000 RPM for $20 \mathrm{~min}$. The supernatant was collected used for IP. Diluted supernatant was added to the equilibrated GFP-Trap magnetic agarose and rotated at $4{ }^{\circ} \mathrm{C}$ overnight. Proteins were eluted with $2 \times$ SDS Laemmli sample buffer and analyzed using SDS-PAGE.

\section{Protein purification and labeling}

aSN was purified from Escherichia coli cells as described previously ${ }^{111}$ Briefly, aSN in a T7-7 plasmid was transformed into BL21(DE3) cells and grown overnight in $10 \mathrm{~mL} \mathrm{LB}$ media with $100 \mu \mathrm{g} / \mathrm{mL}$ ampicillin; the overnight culture was used to inoculate $500 \mathrm{~mL} \mathrm{LB} / 100 \mu \mathrm{g} / \mathrm{mL}$ ampicillin. At $\mathrm{OD}_{600}=0.5-0.6$, protein expression was induced by the addition of $320 \mu \mathrm{M}$ IPTG for $4 \mathrm{~h}$ at $37^{\circ} \mathrm{C}$. The cells were pelleted and resuspended in $25 \mathrm{~mL}$ lysis buffer $(40 \mathrm{mM} \mathrm{NaOH}, 20 \mathrm{mM}$ Tris pH 8.0, $1 \mathrm{mM}$ EDTA, $1 \mathrm{mM}$ phenylmethanesulfonylfluoride (PMSF), $0.1 \%$ Triton X-100) and a complete protease inhibitor cocktail tablet. The lysate was then flash-frozen in liquid nitrogen and stored at $-80^{\circ} \mathrm{C}$ until use. The thawed lysate was treated with $200 \mathrm{U}$ of DNase supplemented with $10 \mathrm{mM} \mathrm{MgCl}$ and $10 \mathrm{mM} \mathrm{CaCl}_{2}$ and incubated for $1 \mathrm{~h}$ at $37^{\circ} \mathrm{C}$ shaking. In all, $10 \mathrm{mM}$ EDTA was added prior to centrifugation at $16,900 \times g$ for $15 \mathrm{~min}$ to remove cellular debris. Two ammonium sulfate cuts $(0.116$ and $0.244 \mathrm{~g} / \mathrm{mL})$ are added to the supernatant, resulting in aSN precipitating in the second step. The pellet was resolubilized in Buffer A ( $25 \mathrm{mM}$ Tris $\mathrm{pH} 8.0,20 \mathrm{mM} \mathrm{NaCl}, 1 \mathrm{mM}$ EDTA) 
with $1 \mathrm{mM}$ PMSF and dialyzed against Buffer $\mathrm{A}$ to remove ammonium sulfate. The supernatant was filtered through a $0.22-\mu \mathrm{m}$ syringe filter and then loaded to an anion exchange column (GE HiTrap Q HP, $5 \mathrm{ml}$ ) and eluted with a gradient to $1 \mathrm{M} \mathrm{NaCl}$. aSN elutes at approximately $300 \mathrm{mM}$ $\mathrm{NaCl}$. Fractions containing aSN were pooled, concentrated, and buffer exchanged into Buffer $\mathrm{C}(25 \mathrm{mM}$ Tris $\mathrm{pH} 8,100 \mathrm{mM} \mathrm{NaCl}, 1 \mathrm{mM}$ EDTA, $0.5 \mathrm{mM}$ TCEP) using Amicon Ultra concentrators (3000 Da MW cutoff). Concentrated samples were once again syringe filtered before loading on a size exclusion column (GE HiLoad 16/600 Superdex75) and eluted at $0.5 \mathrm{~mL} / \mathrm{min}$. Fractions containing aSN were again pooled and concentrated, then stored at $-80^{\circ} \mathrm{C}$.

For site-specific labeling, cysteines were introduced at residues 9 and 72 or 54 and 130. aSN was incubated with $1 \mathrm{mM}$ dithiothreitol for $30 \mathrm{~min}$ and then buffer exchanged into labeling buffer $(20 \mathrm{mM}$ Tris $\mathrm{pH} 7.4,50 \mathrm{mM}$ $\mathrm{NaCl}$, and $6 \mathrm{M}$ guanidine $\mathrm{HCl}$ ). Protein was incubated with the donor fluorophore Alexa Fluor 488 maleimide at a ratio of protein:dye of 1:0.5 for $2 \mathrm{~h}$ at RT with stirring. Then, $4 \times$ molar excess of acceptor fluorophore Alexa Fluor 594 maleimide was added and incubated overnight at $4{ }^{\circ} \mathrm{C}$. Excess dye was removed by buffer exchanging into $20 \mathrm{mM}$ Tris (pH 7.4) and $50 \mathrm{mM} \mathrm{NaCl}$ using Amicon concentrators and two tandem HiTrap Desalting Columns. Labeled proteins were aliquoted, snap frozen, and stored at $-80^{\circ} \mathrm{C}$ until use.

\section{Pre-formed fibrils}

Bulk PFFs were prepared following the protocol developed by Virginia Lee's group ${ }^{58}$. Briefly, aliquots of recombinant $\mathrm{aSN}(\mathrm{m})$ were rapidly thawed and spun at $25,000 \times g$ for $30 \mathrm{~min}$ at $4^{\circ} \mathrm{C}$. For bulk PFF production, approximately $500 \mu \mathrm{L}$ of $5 \mathrm{mg} / \mathrm{mL}$ monomeric protein was added to a 2-mL Eppendorf tube and placed on a thermomixer-C (Eppendorf, USA) shaker and incubated at $37^{\circ} \mathrm{C}$ and 1000 RPM for up to $144 \mathrm{~h}$. Every $6 \mathrm{~h}, 5 \mu \mathrm{L}$ aliquots were removed and assayed for ThT fluorescence until a plateau in aggregation was observed and the tube was opaque. Twenty microliters of aliquots of $5 \mathrm{mg} / \mathrm{mL}$ PFF were then flash frozen and stored at $-80^{\circ} \mathrm{C}$.

\section{aSN oligomerization and cross-linking assay}

In vitro recombinant aSN oligomerization assay was adapted from the methods described by Otzen et al. ${ }^{90}$. In brief, aliquots of purified recombinant aSN were quick-thawed and centrifuged at $25,000 \times g$ for $30 \mathrm{~min}$ at $4{ }^{\circ} \mathrm{C}$. The top $95 \%$ of supernatant was removed and pooled. Concentration was determined using UV280 absorbance on a Spectromaxi3x (Molecular Devices) and the protein was diluted to a final concentration of $5 \mathrm{mg} / \mathrm{mL}(\sim 350 \mu \mathrm{M})$. Aliquots of $150 \mu \mathrm{L}$ were dispensed into $2-\mathrm{mL}$ lowprotein-binding centrifuge tubes and compounds were added to each tube for a final concentration of $70 \mu \mathrm{M}$ (1:5 compound:protein ratio). Samples were then incubated under continuous shaking (900 RPM) at $37^{\circ} \mathrm{C}$ for $12 \mathrm{~h}$ (ThermoMixer-C, Eppendorf, Hauppauge, NY).

After oligomerization, a $50-\mu \mathrm{L}$ aliquot was removed and cross-linked following the protocol developed by Jensen et al. ${ }^{91}$. In brief, oligomer aliquot was incubated with $1.6 \%$ PFA in PBS for $1 \mathrm{~h}$ at ambient temperature. Cross-linking was quenched with tenfold excess Tris $(\mathrm{pH}$ 7.4) and equal volume was prepared in $4 \times$ Laemmli loading buffer (BioRad) for subsequent SDS-PAGE, transfer, and immunoblot analysis as previously detailed above.

A 10- $\mu \mathrm{L}$ aliquot from each sample was taken and diluted with $290 \mu \mathrm{L}$ ThT working solution (100 $\mu \mathrm{M}$ ThT in PBS) and dispensed into 3 wells of a 384well, black opaque plate. Additional samples of PBS, aSN $(m)$, and aSN PFF were used as controls. Plates were incubated at ambient temperature for $10 \mathrm{~min}$ and then fluorescence read at $450 \mathrm{ex} / 490 \mathrm{em}$ on a Spectromax-i3x (Molecular Devices).

\section{ThT aggregation assays: non-seeded, seeded, and cellular homogenate}

ThT (Sigma, product no. T3516) was dissolved in PBS buffer and was filtered through a $0.2-\mu \mathrm{m}$ syringe filter to make a stock solution of $2.5 \mathrm{mM}$. Working ThT concentration was $20 \mu \mathrm{M}$. PFF seeds were produced via constant agitation of 1000 RPM at $37^{\circ} \mathrm{C}$ (ThermoMixer-C, Eppendorf, Hauppauge, NY) of $500 \mu \mathrm{L}$ of $350 \mu \mathrm{M}$ aSN in PBS in a $2 \mathrm{~mL}$ tube for 5 days. PFFs were aliquoted and flash frozen and stored at $-80^{\circ} \mathrm{C}$ until further use. PFF seeds were rapidly thawed, diluted to $35 \mu \mathrm{M}$ in PBS, and sonicated 120 pulses at $50 \%$ using a probe-tip sonicator following the established protocols ${ }^{58}$. Master mix samples for each of the different ThT assays were prepared and dispensed in triplicate as $50 \mu \mathrm{L}$ in 384-well flat, black-bottom polypropylene plates (PN 781209, Greiner Bio-One) and sealed with fluorescently transparent film. Conditions for non-seeded, seeded, and cell homogenate assays varied slightly.

Non-seeded ThT. Non-seeded ThT master mix was prepared in cell culture PBS with $140 \mu \mathrm{M}(2 \mathrm{mg} / \mathrm{mL})$ aSN $(\mathrm{m})$ and dispensed into compound plates at $70 \mu \mathrm{M}$ final compound concentration (1:2 compound:protein ratio).

Seeded ThT. Seeded ThT experiments were performed at a lower monomer concentration to preclude spontaneous aSN aggregation during the time course of the experiment. For both seeded and cell homogenate experiments, sample were prepared in PBS $+20 \mu \mathrm{M}$ ThT with or without $15 \mu \mathrm{M}$ aSN $(\mathrm{m})+/-5 \%$ PFF $(0.75 \mu \mathrm{M})$. Additional control samples of $15 \mu \mathrm{M}$ PFF and $0.75 \mu \mathrm{M}$ PFF were also prepared. All empty wells were filled with PBS for a thermal sink and to help prevent evaporation. Assay plates were prepared as pre-cellular FRET dose response above. Dose response was done across 8 concentrations $(10 \mathrm{nM}$ to $50 \mu \mathrm{M})$. The assay was run at $37^{\circ} \mathrm{C}$ with mild shaking (200 RPM) in a Cytation3 plate reader. The ThT fluorescence was monitored with excitation filter of $450 \mathrm{~nm}$ and emission filter of $490 \mathrm{~nm}$. Readings were acquired every $20 \mathrm{~min}$ for a minimum of $24 \mathrm{~h}$. Seeded ThT aggregation $I C_{50}$ values were determined by first normalizing all ThT curves to the maximal signal at plateau for the aSN + $5 \%$ PFF + DMSO samples. The normalized fluorescence at each concentration was fit to the Hill equation to resolve an effective $I_{50}$ of seeded aggregation inhibition.

Cell homogenate ThT. Cell homogenate ThT experiments were performed with inter-protomeric aSN biosensors expressed in HEK293 cells as described above. Cells were homogenized in native lysis buffer with proteasome inhibitor. After lysis, cells were centrifuged at $16,000 \times g$ for $1 \mathrm{~min}$ and total protein concentration of the supernatant was quantified via BCA analysis. Cell lysates from the three different sets of plasmids, along with an untransfected HEK293 control, were combined with PBS and ThT $(20 \mu \mathrm{M})$ to reach a $5 \%$ lysate seeded solution. A second set of test samples were combined with PBS, ThT, and $15 \mu \mathrm{M}$ aSN(m). PBS only was used as background, with 5\% PFF or aSyn $(\mathrm{m})$ as negative control and $5 \%$ PFF with aSyn $(\mathrm{m})$ as positive control. Samples were plated in triplicate in 384-well flat, black-bottom polypropylene plates (PN 781209, Greiner BioOne) and sealed with fluorescently transparent film. ThT fluorescence was monitored using a Cytation 3 (excitation: $450 \mathrm{~nm}$, emission: $490 \mathrm{~nm}$ ) under continuous shaking with reads every $10 \mathrm{~min}$ for $48 \mathrm{~h}$.

\section{SMFRET measurements}

Single-molecule FRET measurements were carried out using $\sim 30 \mathrm{pM}$ of labeled aSN in $20 \mathrm{mM}$ Tris pH 7.4 and $50 \mathrm{mM} \mathrm{NaCl}$ in 8-chambered Nunc coverslips (ThermoFisher) passivated with poly(ethylene glycol) poly(Llysine) to reduce protein adsorption to the chambers. Measurements were made on a MicroTime 200 time-resolved confocal microscope (Picoquant) as described previously ${ }^{112}$. Laser power was adjusted to $\sim 30 \mu \mathrm{W}$ before sample illumination. Fluorescence emission was collected through the objective and passed through a $150-\mu \mathrm{m}$ diameter pinhole. Photons were separated by an HQ585LP dichroic in combination with ET525/50M and HQ600LP filters and detected by avalanche photodiodes. Photon traces were collected in 1-ms time bins for $1 \mathrm{~h}$. A cutoff of 25 counts/ms was applied to discriminate between bursts arising from fluorescently labeled protein and background noise. The FRET efficiency $\left(E_{\text {eff }}\right)$ was calculated using the SymphoTime 64 software. SmFRET histograms were fit with Gaussian distributions to determine the peak $E_{\text {eff }}$ values. Each measurement was carried out three times and mean $E_{\text {eff }}$ values are the average and SD of these measurements (Supplementary Table 2). Alignment of instrument and analysis were verified using smFRET reference standards consisting of 10, 14, and 18 base pair dsDNA labeled with Alexa 488 and Alex 594 with their $5^{\prime}$ and $3^{\prime}$ ends.

\section{Protein-observed fluorine NMR (PrOF NMR)}

Experiments were run on a Bruker 600-MHz Avance NEO (6001), equipped with a $5 \mathrm{~mm}$ triple resonance cryoprobe. $3 \mathrm{FY}$-aSN was diluted in $\mathrm{PBS}, \mathrm{pH}=$ 7.4 buffer by the addition of $\mathrm{D}_{2} \mathrm{O}$ and $0.1 \%$ trifluoroacetic acid (TFA) to final concentrations of 5 and $0.07 \%$, respectively, in a total NMR sample volume of $150 \mu \mathrm{L}$. Two one-dimensional ${ }^{19} \mathrm{~F}$ NMR spectra were taken of the protein sample at an 01P of $-75 \mathrm{ppm}, \mathrm{NS}=16, \mathrm{D} 1=1 \mathrm{~s}$, and $\mathrm{AQ}=0.5 \mathrm{~s}$ (TFA reference set to $-75.25 \mathrm{ppm}$ ) and an O1P of $-136 \mathrm{ppm}, \mathrm{NS}=3072, \mathrm{D} 1=$ $0.6 \mathrm{~s}$, and $\mathrm{AQ}=0.05 \mathrm{~s}$ (protein resonances). Ligand stock solutions of $1-10 \mathrm{mM}$ were prepared in DMSO and titrated into the protein solutions 
$(20 \mu \mathrm{M})$. As a control experiment, in a separate NMR tube, DMSO was added to a final concentration of $0.5,0.6,0.8,1.5$, and $2.5 \%$ to control for any solvent-induced chemical shifts. For each concentration, a TFA reference spectrum and a protein $1 \mathrm{D}-{ }^{19} \mathrm{~F}$ NMR spectrum were acquired.

\section{Statistical analysis}

Data are shown as mean \pm SD unless stated otherwise. Statistical analysis for FLT and FRET experiments were conducted by an unpaired Student's $t$ test using GraphPad to determine statistical significance for all experiments. Values of $p$ value $<0.05$ were considered statistically significant.

Outcome measures for PFF-induced neuron pathology (DxA 81A/NeuN ratio) were compared between experimental conditions using linear mixed-effects models, with fixed effects for condition and random effects for experiment, well by condition, and experiment by well by condition, to account for within-well correlation, variation by experiment, and variation of conditions across experiments. Outcome measures were logtransformed for analysis for approximate normality and homogeneity of variance across conditions and experiments. Estimated differences between conditions, along with their 95\% confidence intervals, were exponentiated to report as ratios of the outcome measures between conditions. Analyses were conducted using $\mathrm{R}^{113}$ version 3.6 .1 , including the packages Ime $4^{114}$ version 1.1-21 and ImerTest ${ }^{115}$ version 3.1-1.

\section{Reporting summary}

Further information on research design is available in the Nature Research Reporting Summary linked to this article.

\section{DATA AVAILABILITY}

The datasets generated and/or analyzed during the current study are available from the corresponding author on reasonable request.

\section{CODE AVAILABILITY}

Analyses for the FLT/SUPR HTS data were performed with custom scripts for waveform fitting and spectral unmixing (Matlab 2019a) and Hit identification (Excel 2016). Analyses for the primary neuron pathology assay were conducted using $R$ (version 3.6.1), including the packages Ime $4^{114}$ version $1.1-21$ and ImerTest ${ }^{115}$ version 3.1-1. Scripts are available from the corresponding author on reasonable request.

Received: 18 June 2020; Accepted: 2 June 2021; Published online: 28 June 2021

\section{REFERENCES}

1. Power, J. H., Barnes, O. L. \& Chegini, F. Lewy bodies and the mechanisms of neuronal cell death in Parkinson's disease and dementia with lewy bodies. Brain Pathol. https://doi.org/10.1111/bpa.12344 (2015).

2. Uversky, V. N. \& Eliezer, D. Biophysics of Parkinson's disease: structure and aggregation of alpha-synuclein. Curr. Protein Pept. Sci. 10, 483-499 (2009).

3. Gallardo, G., Schlüter, O. M. \& Südhof, T. C. A molecular pathway of neurodegeneration linking alpha-synuclein to ApoE and Abeta peptides. Nat. Neurosci. 11, 301-308 (2008).

4. Lorenzen, N. et al. The role of stable a-synuclein oligomers in the molecular events underlying amyloid formation. J. Am. Chem. Soc. 136, 3859-3868 (2014).

5. Uversky, V. N. Alpha-synuclein misfolding and neurodegenerative diseases. Curr. Protein Pept. Sci. 9, 507-540 (2008).

6. Emadi, S. et al. Inhibiting aggregation of alpha-synuclein with human single chain antibody fragments. Biochemistry 43, 2871-2878 (2004).

7. Wirths, O. et al. Lewy body variant of Alzheimer's disease: alpha-synuclein in dystrophic neurites of A beta plaques. Neuroreport 11, 3737-3741 (2000).

8. Spillantini, M. G., Crowther, R. A., Jakes, R., Hasegawa, M. \& Goedert, M. alphasynuclein in filamentous inclusions of Lewy bodies from Parkinson's disease and dementia with Lewy bodies. Proc. Natl Acad. Sci. USA 95, 6469-6473 (1998).

9. Rockenstein, E. et al. Accumulation of oligomer-prone a-synuclein exacerbates synaptic and neuronal degeneration in vivo. Brain 137, 1496-1513 (2014).

10. Iljina, M. et al. Nanobodies raised against monomeric a-synuclein inhibit fibril formation and destabilize toxic oligomeric species. BMC Biol. 15, 57 (2017).
11. Lam, H. T., Graber, M. C., Gentry, K. A. \& Bieschke, J. Stabilization of a-synuclein fibril clusters prevents fragmentation and reduces seeding activity and toxicity. Biochemistry 55, 675-685 (2016).

12. Celej, M. S. et al. Toxic prefibrillar a-synuclein amyloid oligomers adopt a distinctive antiparallel $\beta$-sheet structure. Biochem. J. 443, 719-726 (2012).

13. Colla, E. et al. Accumulation of toxic a-synuclein oligomer within endoplasmic reticulum occurs in a-synucleinopathy in vivo. J. Neurosci. 32, 3301-3305 (2012).

14. He, X. L., Giurleo, J. T. \& Talaga, D. S. Role of small oligomers on the amyloidogenic aggregation free-energy landscape. J. Mol. Biol. 395, 134-154 (2010).

15. Shahmoradian, S. H. et al. Lewy pathology in Parkinson's disease consists of crowded organelles and lipid membranes. Nat. Neurosci. 22, 1099-1109 (2019).

16. Imberdis, T. et al. Cell models of lipid-rich a-synuclein aggregation validate known modifiers of a-synuclein biology and identify stearoyl-CoA desaturase. Proc. Natl Acad. Sci. USA 116, 20760-20769 (2019).

17. Galvagnion, $C$. et al. Chemical properties of lipids strongly affect the kinetics of the membrane-induced aggregation of a-synuclein. Proc. Natl Acad. Sci. USA 113, 7065-7070 (2016).

18. Kim, S. et al. GBA1 deficiency negatively affects physiological a-synuclein tetramers and related multimers. Proc. Natl Acad. Sci. USA 115, 798-803 (2018).

19. Dettmer, U. et al. Parkinson-causing a-synuclein missense mutations shift native tetramers to monomers as a mechanism for disease initiation. Nat. Commun. 6 , 7314-7314 (2015).

20. Luth, E. S., Bartels, T., Dettmer, U., Kim, N. C. \& Selkoe, D. J. Purification of alphasynuclein from human brain reveals an instability of endogenous multimers as the protein approaches purity. Biochemistry 54, 279-292 (2015).

21. Pochapsky, T. C. From intrinsically disordered protein to context-dependent folding: The a-synuclein tetramer is teased out of hiding. Proc. Natl Acad. Sci. USA 112, 9502-9503 (2015).

22. Dettmer, U., Newman, A. J., von Saucken, V. E., Bartels, T. \& Selkoe, D. KTKEGV repeat motifs are key mediators of normal a-synuclein tetramerization: their mutation causes excess monomers and neurotoxicity. Proc. Natl Acad. Sci. USA 112, 9596-9601 (2015).

23. Wang, W. et al. A soluble a-synuclein construct forms a dynamic tetramer. Proc. Natl Acad. Sci. USA 108, 17797-17802 (2011).

24. Bartels, T., Choi, J. G. \& Selkoe, D. J. alpha-Synuclein occurs physiologically as a helically folded tetramer that resists aggregation. Nature https://doi.org/ 10.1038/nature10324 (2011).

25. Sangwan, S. et al. Inhibition of synucleinopathic seeding by rationally designed inhibitors. Elife https://doi.org/10.7554/eLife.46775 (2020).

26. Pujols, J. et al. High-throughput screening methodology to identify alphasynuclein aggregation inhibitors. Int. J. Mol. Sci. https://doi.org/10.3390/ ijms18030478 (2017).

27. Pujols, J. et al. Small molecule inhibits a-synuclein aggregation, disrupts amyloid fibrils, and prevents degeneration of dopaminergic neurons. Proc. Natl Acad. Sci. USA 115, 10481-10486 (2018).

28. Gao, X. et al. Human Hsp70 disaggregase reverses Parkinson's-linked a-synuclein amyloid fibrils. Mol. Cell 59, 781-793 (2015).

29. Carija, A. et al. Biasing the native a-synuclein conformational ensemble towards compact states abolishes aggregation and neurotoxicity. Redox Biol. 22, 101135 (2019).

30. Chen, S. W. et al. Structural characterization of toxic oligomers that are kinetically trapped during a-synuclein fibril formation. Proc. Natl Acad. Sci. USA https:// doi.org/10.1073/pnas.1421204112 (2015).

31. Deas, E. et al. Alpha-synuclein oligomers interact with metal ions to induce oxidative stress and neuronal death in Parkinson's disease. Antioxid. Redox Signal. 24, 376-391 (2016).

32. Carmo-Gonçalves, P., Pinheiro, A. S., Romão, L., Cortines, J. \& Follmer, C. UVinduced selective oxidation of Met5 to Met-sulfoxide leads to the formation of neurotoxic fibril-incompetent a-synuclein oligomers. Amyloid 21, 163-174 (2014).

33. Lorenzen, $\mathrm{N}$. et al. How epigallocatechin gallate can inhibit a-synuclein oligomer toxicity in vitro. J. Biol. Chem. 289, 21299-21310 (2014).

34. Giehm, L., Lorenzen, N. \& Otzen, D. E. Assays for a-synuclein aggregation. Methods 53, 295-305 (2011).

35. Herva, M. E. et al. Anti-amyloid compounds inhibit a-synuclein aggregation induced by protein misfolding cyclic amplification (PMCA). J. Biol. Chem. 289, 11897-11905 (2014).

36. Perni, M. et al. A natural product inhibits the initiation of a-synuclein aggregation and suppresses its toxicity. Proc. Natl Acad. Sci. USA 114, E1009-E1017 (2017).

37. Kurnik, M. et al. Potent a-synuclein aggregation inhibitors, identified by highthroughput screening, mainly target the monomeric state. Cell Chem. Biol. 25, 1389-1402 (2018). 
38. Li, X. et al. Naturally occurring antibodies isolated from PD patients inhibit synuclein seeding in vitro and recognize Lewy pathology. Acta Neuropathol. 137, 825-836 (2019).

39. Weihofen, A. et al. Development of an aggregate-selective, human-derived asynuclein antibody BIIB054 that ameliorates disease phenotypes in Parkinson's disease models. Neurobiol. Dis. 124, 276-288 (2019).

40. Schofield, D. J. et al. Preclinical development of a high affinity a-synuclein antibody, MEDI1341, that can enter the brain, sequester extracellular a-synuclein and attenuate a-synuclein spreading in vivo. Neurobiol. Dis. 132, 104582 (2019).

41. Wagner, J. et al. Anle138b: a novel oligomer modulator for disease-modifying therapy of neurodegenerative diseases such as prion and Parkinson's disease. Acta Neuropathol. 125, 795-813 (2013).

42. Moussaud, S. et al. Targeting a-synuclein oligomers by protein-fragment complementation for drug discovery in synucleinopathies. Expert Opin. Ther. Targets 19, 589-603 (2015)

43. Gruber, S. et al. Discovery of enzyme modulators via high-throughput timeresolved FRET in living cells. J. Biomol. Screen. 19, 1-9 (2014).

44. Outeiro, T. F. et al. Formation of toxic oligomeric alpha-synuclein species in living cells. PLOS ONE 3, e1867 (2008)

45. Schaaf, T. M. et al. High-throughput spectral and lifetime-based FRET screening in living cells to identify small-molecule effectors of SERCA. SLAS Discov. 22, 262-273 (2017)

46. Lo, C. H. et al. An innovative high-throughput screening approach for discovery of small molecules that inhibit TNF receptors. SLAS Discov. 22, 950-961 (2017).

47. Lo, C. H. et al. Targeting the ensemble of heterogeneous tau oligomers in cells: a novel small molecule screening platform for tauopathies. Alzheimers Dis. Dementia 15, 1489-1502 (2019).

48. Lo, C. H., Huber, E. C. \& Sachs, J. N. Conformational states of TNFR1 as a molecular switch for receptor function. Protein Sci. https://doi.org/10.1002/ pro.3829 (2020).

49. Lo, C. H. et al. Noncompetitive inhibitors of TNFR1 probe conformational activation states. Sci. Signal. https://doi.org/10.1126/scisignal.aav5637 (2019).

50. Schaaf, T. M. et al. Red-shifted FRET biosensors for high-throughput fluorescence lifetime screening. Biosensors https://doi.org/10.3390/bios8040099 (2018).

51. Stroik, D. R. et al. Targeting protein-protein interactions for therapeutic discovery via FRET-based high-throughput screening in living cells. Sci. Rep. 8, 12560 (2018).

52. Rebbeck, R. T. et al. High-throughput screens to discover small-molecule modulators of ryanodine receptor calcium release channels. J. Biomol. Screen. https://doi.org/10.1177/1087057116674312 (2016).

53. Perrin, R. J. et al. Epitope mapping and specificity of the anti-a-synuclein monoclonal antibody Syn-1 in mouse brain and cultured cell lines. Neurosci. Lett. 349, 133-135 (2003)

54. Jakes, R. et al. Epitope mapping of LB509, a monoclonal antibody directed against human a-synuclein. Neurosci. Lett. 269, 13-16 (1999).

55. Lee, M. K. et al. Human alpha-synuclein-harboring familial Parkinson's diseaselinked Ala-53 -> Thr mutation causes neurodegenerative disease with alphasynuclein aggregation in transgenic mice. Proc. Natl Acad. Sci. USA 99, 8968-8973 (2002).

56. Zhou, H. et al. Tetramethylpyrazine analogue T-006 promotes the clearance of alpha-synuclein by enhancing proteasome activity in Parkinson's disease models. Neurotherapeutics https://doi.org/10.1007/s13311-019-00759-8 (2019).

57. Cantuti-Castelvetri, I. et al. Alpha-synuclein and chaperones in dementia with Lewy bodies. J. Neuropathol. Exp. Neurol. 64, 1058-1066 (2005).

58. Volpicelli-Daley, L. A., Luk, K. C. \& Lee, V. M. Y. Addition of exogenous a-synuclein preformed fibrils to primary neuronal cultures to seed recruitment of endogenous a-synuclein to Lewy body and Lewy neurite-like aggregates. Nat. Protoc. 9, 2135-2146 (2014).

59. Volpicelli-Daley, L. A. et al. Exogenous a-synuclein fibrils induce Lewy body pathology leading to synaptic dysfunction and neuron death. Neuron $\mathbf{7 2}, 57-71$ (2011).

60. Mohite, G. M. et al. Comparison of kinetics, toxicity, oligomer formation, and membrane binding capacity of a-synuclein familial mutations at the A53 site, including the newly discovered A53V mutation. Biochemistry 57, 5183-5187 (2018).

61. Flagmeier, P. et al. Mutations associated with familial Parkinson's disease alter the initiation and amplification steps of a-synuclein aggregation. Proc. Natl Acad. Sci. USA 113, 10328-10333 (2016).

62. Sierecki, E. et al. Nanomolar oligomerization and selective co-aggregation of asynuclein pathogenic mutants revealed by single-molecule fluorescence. Sci. Rep. 6, 37630 (2016).

63. Lemkau, L. R. et al. Site-specific perturbations of alpha-synuclein fibril structure by the Parkinson's disease associated mutations A53T and E46K. PLOS ONE https://doi.org/10.1371/journal.pone.0049750 (2013).
64. Kang, L., Wu, K.-P., Vendruscolo, M. \& Jean B. The A53T mutation is key in defining the differences in the aggregation kinetics of human and mouse asynuclein. J. Am. Chem. Soc. 133, 13465-13470 (2011).

65. Tosatto, L. et al. Single-molecule FRET studies on alpha-synuclein oligomerization of Parkinson's disease genetically related mutants. Sci. Rep. 5, 16696 (2015).

66. Schaaf, T. M., Peterson, K. C., Grant, B. D., Thomas, D. D. \& Gillispie, G. D. Spectral unmixing plate reader: high-throughput, high-precision FRET assays in living cells. J. Biomol. Screen. https://doi.org/10.1177/1087057116679637 (2016).

67. Outeiro, T. F. et al. Sirtuin 2 inhibitors rescue alpha-synuclein-mediated toxicity in models of Parkinson's disease. Science 317, 516-519 (2007).

68. Harrison, I. F., Smith, A. D. \& Dexter, D. T. Pathological histone acetylation in Parkinson's disease: Neuroprotection and inhibition of microglial activation through SIRT 2 inhibition. Neurosci. Lett. 666, 48-57 (2018).

69. Xicoy, H., Wieringa, B. \& Martens, G. J. The SH-SY5Y cell line in Parkinson's disease research: a systematic review. Mol. Neurodegener. 12, 10 (2017).

70. Khalaf, O. et al. The $\mathrm{H} 50 \mathrm{Q}$ mutation enhances a-synuclein aggregation, secretion, and toxicity. J. Biol. Chem. 289, 21856-21876 (2014).

71. Tofaris, G. K., Layfield, R. \& Spillantini, M. G. alpha-Synuclein metabolism and aggregation is linked to ubiquitin-independent degradation by the proteasome. FEBS Lett. 509, 22-26 (2001).

72. Pandey, N., Schmidt, R. E. \& Galvin, J. E. The alpha-synuclein mutation E46K promotes aggregation in cultured cells. Exp. Neurol. 197, 515-520 (2006).

73. Turriani, E. et al. Treatment with diphenyl-pyrazole compound anle138b/c reveals that a-synuclein protects melanoma cells from autophagic cell death. Proc. Natl Acad. Sci. USA 114, E4971-E4977 (2017)

74. Jiang, S. X. et al. Chlortetracycline and demeclocycline inhibit calpains and protect mouse neurons against glutamate toxicity and cerebral ischemia. J. Biol. Chem. 280, 33811-33818 (2005).

75. Shams, R., Banik, N. L. \& Haque, A. Calpain in the cleavage of alpha-synuclein and the pathogenesis of Parkinson's disease. Prog. Mol. Biol. Transl. Sci. 167, 107-124 (2019).

76. Hassen, G. W. et al. Effects of novel calpain inhibitors in transgenic animal model of Parkinson's disease/dementia with Lewy bodies. Sci. Rep. 8, 18083 (2018).

77. Xu, B. et al. Inhibition of calpain prevents manganese-induced cell injury and alpha-synuclein oligomerization in organotypic brain slice cultures. PLOS ONE 10, e0119205 (2015).

78. Diepenbroek, M. et al. Overexpression of the calpain-specific inhibitor calpastatin reduces human alpha-Synuclein processing, aggregation and synaptic impairment in [A30P]aSyn transgenic mice. Hum. Mol. Genet. 23, 3975-3989 (2014).

79. Games, D. et al. Reducing C-terminal-truncated alpha-synuclein by immunotherapy attenuates neurodegeneration and propagation in Parkinson's disease-like models. J. Neurosci. 34, 9441-9454 (2014).

80. Czapski, G. A., Gąssowska, M., Wilkaniec, A., Cieślik, M. \& Adamczyk, A. Extracellular alpha-synuclein induces calpain-dependent overactivation of cyclindependent kinase 5 in vitro. FEBS Lett. 587, 3135-3141 (2013).

81. Burré, J., Sharma, M. \& Südhof, T. C. Cell biology and pathophysiology of asynuclein. Cold Spring Harb. Perspect. Med. https://doi.org/10.1101/cshperspect. a024091 (2018).

82. Kanaan, N. M. \& Manfredsson, F. P. Loss of functional alpha-synuclein: a toxic event in Parkinson's disease. J. Parkinsons Dis. 2, 249-267 (2012).

83. Harischandra, D. S., Jin, H., Anantharam, V., Kanthasamy, A. \& Kanthasamy, A. G. a-Synuclein protects against manganese neurotoxic insult during the early stages of exposure in a dopaminergic cell model of Parkinson's disease. Toxicol. Sci. 143, 454-468 (2015).

84. Kanthasamy, A. et al. Emerging neurotoxic mechanisms in environmental factors-induced neurodegeneration. Neurotoxicology 33, 833-837 (2012).

85. Benskey, M. J., Perez, R. G. \& Manfredsson, F. P. The contribution of alpha synuclein to neuronal survival and function - implications for Parkinson's disease. J. Neurochem. 137, 331-359 (2016).

86. Chandra, S., Gallardo, G., Fernández-Chacón, R., Schlüter, O. M. \& Südhof, T. C. Alpha-synuclein cooperates with CSPalpha in preventing neurodegeneration. Cell 123, 383-396 (2005)

87. Kragh, C. L. et al. Alpha-synuclein aggregation and Ser-129 phosphorylationdependent cell death in oligodendroglial cells. J. Biol. Chem. 284, 10211-10222 (2009).

88. Okochi, M. et al. Constitutive phosphorylation of the Parkinson's disease associated alpha-synuclein. J. Biol. Chem. 275, 390-397 (2000).

89. Li, C. et al. $19 \mathrm{~F} \mathrm{NMR}$ studies of alpha-synuclein conformation and fibrillation. Biochemistry 48, 8578-8584 (2009).

90. Paslawski, W., Lorenzen, N. \& Otzen, D. E. Formation and characterization of asynuclein oligomers. Methods Mol. Biol. 1345, 133-150 (2016).

91. Ruesink, H. et al. Stabilization of a-synuclein oligomers using formaldehyde. PLOS ONE 14, e0216764 (2019). 
92. Wördehoff, M. M. et al. Single fibril growth kinetics of a-synuclein. J. Mol. Biol. 427, 1428-1435 (2015).

93. Iljina, M. et al. Kinetic model of the aggregation of alpha-synuclein provides insights into prion-like spreading. Proc. Natl Acad. Sci. USA 113, E1206-E1215 (2016).

94. Pinotsi, D., Buell, A. K., Dobson, C. M., Kaminski Schierle, G. S. \& Kaminski, C. F. A label-free, quantitative assay of amyloid fibril growth based on intrinsic fluorescence. Chembiochem 14, 846-850 (2013).

95. Fink, A. L. The aggregation and fibrillation of alpha-synuclein. Acc. Chem. Res. 39, 628-634 (2006).

96. Saha, S., Khan, M. A. I., Mudhara, D. \& Deep, S. Tuning the balance between fibrillation and oligomerization of a-synuclein in the presence of dopamine. ACS Omega 3, 14213-14224 (2018).

97. De Oliveira, G. A. P. \& Silva, J. L. Alpha-synuclein stepwise aggregation reveals features of an early onset mutation in Parkinson's disease. Commun. Biol. https:// doi.org/10.1038/s42003-019-0598-9 (2019).

98. Guerrero-Ferreira, R. et al. Cryo-EM structure of alpha-synuclein fibrils. Elife https://doi.org/10.7554/eLife.36402 (2018).

99. Li, B. et al. Cryo-EM of full-length a-synuclein reveals fibril polymorphs with a common structural kernel. Nat. Commun. 9, 3609 (2018).

100. Romo, T. D., Lewis, A. K., Braun, A. R., Grossfield, A. \& Sachs, J. N. Minimal nucleation state of a-synuclein is stabilized by dynamic threonine-water networks. ACS Chem. Neurosci. 8, 1859-1864 (2017).

101. Tuttle, M. D. et al. Solid-state NMR structure of a pathogenic fibril of full-length human alpha-synuclein. Nat. Struct. Mol. Biol. 23, 1-9 (2016).

102. Rodriguez, J. A. et al. Structure of the toxic core of a-synuclein from invisible crystals. Nature 525, 486-490 (2015).

103. Theillet, F. X. et al. Structural disorder of monomeric a-synuclein persists in mammalian cells. Nature 530, 45-50 (2016).

104. Newberry, R. W., Leong, J. T., Chow, E. D., Kampmann, M. \& DeGrado, W. F. Deep mutational scanning reveals the structural basis for a-synuclein activity. Nat. Chem. Biol. https://doi.org/10.1038/s41589-020-0480-6 (2020).

105. Froula, J. M. et al. Defining a-synuclein species responsible for Parkinson's disease phenotypes in mice. J. Biol. Chem. 294, 10392-10406 (2019).

106. Bohrmann, B. et al. Self-assembly of beta-amyloid 42 is retarded by small molecular ligands at the stage of structural intermediates. J. Struct. Biol. 130, 232-246 (2000)

107. González-Lizárraga, F. et al. Repurposing doxycycline for synucleinopathies: remodelling of a-synuclein oligomers towards non-toxic parallel beta-sheet structured species. Sci. Rep. 7, 41755 (2017).

108. Woerman, A. L. et al. Propagation of prions causing synucleinopathies in cultured cells. Proc. Natl Acad. Sci. USA 112, E4949-E4958 (2015).

109. Yang, F., Moss, L. G. \& Phillips, G. N. Jr The molecular structure of green fluorescent protein. Nat. Biotechnol. 14, 1246-1251 (1996).

110. Zhang, J. H., Chung, T. D. \& Oldenburg, K. R. A simple statistical parameter for use in evaluation and validation of high throughput screening assays. J. Biomol. Screen. 4, 67-73 (1999).

111. Trexler, A. J. \& Elizabeth, R. N-terminal acetylation is critical for forming alphahelical oligomer of alpha-synuclein. Protein Sci. 21, 601-605 (2012).

112. Wickramasinghe, S. P. \& Rhoades, E. Measuring interactions between tau and aggregation inducers with single-molecule Förster resonance energy transfer. Methods Mol. Biol. 2141, 755-775 (2020).

113. $\mathrm{R}$ Core Team. R: A Language and Environment for Statistical Computing ( $\mathrm{R}$ Foundation for Statistical Computing, 2019).

114. Bates, D., Mächler, M., Bolker, B. \& Walker, S. Fitting linear mixed-effects models using Ime4. J. Stat. Softw. https://doi.org/10.18637/jss.v067.i01 (2015).

115. Kuznetsova, A., Brockhoff, P. B. \& Christensen, R. H. B. ImerTest Package: tests in linear mixed effects models. J. Stat. Softw. https://doi.org/10.18637/jss.v082.i13 (2017).

\section{ACKNOWLEDGEMENTS}

We thank Dr. Nagamani Vunnam from the Sachs Group; Dr. Tory Schaaf, Samantha Yuen, and Dr. Andrew R. Thompson from the Thomas Group; Benjamin Grant from Fluorescence Innovations; and Dr. Robyn T. Rebecck for technical support and discussions. Compound dispensing was performed at the UMN Institute of
Therapeutic Drug Discovery and Development (ITDD) High-Throughput Screening Laboratory. Spectroscopy was performed at the UMN Biophysical Technology Center and at the adjacent Photonic Pharma laboratory. This research uses technology patented by the University of Minnesota, with an exclusive commercial license to Photonic Pharma LLC. The authors disclose receipt of the following financial support for the research, authorship, and/or publication of this article: this study was supported by U.S. National Institutes of Health (NIH) grants to J.N.S. and W.C.K.P. (NINDS 1R01NS117968 and NINDS R21 NS109505) and to J.N.S. and D.D.T. (NIA SBIR 1R43AG063675). This research was also supported by the NIH National Center for Advancing Translational Sciences, grant UL1TR002494. Support was also provided by T32GM132039 to K.A. and RF1AG053951 to E.R. The content is solely the responsibility of the authors and does not necessarily represent the official views of the National Institutes of Health's National Center for Advancing Translational Sciences.

\section{AUTHOR CONTRIBUTIONS}

A.R.B. and J.N.S. conceived of and directed the study. A.R.B. designed and conducted the experiments. E.E.L. provided assistance with cell-based assays, conducted western blot and immunoprecipitation experiments, and provided comments and edits to the manuscript; M.C.Y. produced and purified recombinant protein; N.N.K. conducted cell lysate seeded aggregation assays; K.A. and E.R. performed recombinant protein labeling and smFRET experiments; P.K. and W.C.K.P. performed PrOF NMR experiments; M.H. and K.L. performed primary neuron PFF assays; M.E. and R.B. performed statistical model development and analysis on the PFF pathology model C.H.L., D.D.T., and R.L.C provided expertise on FRET and HTS and provided comments and edits to the manuscript; A.R.B. and J.N.S. wrote the manuscript.

\section{COMPETING INTERESTS}

D.D.T. and R.L.C. hold equity in and serve as executive officers for Photonic Pharma LLC, a company that owns intellectual property related to technology used in part of this project. These relationships have been reviewed and managed by the University of Minnesota in accordance with its conflict of interest polices. The remaining authors declare no competing interests.

\section{ADDITIONAL INFORMATION}

Supplementary information The online version contains supplementary material available at https://doi.org/10.1038/s41531-021-00195-6.

Correspondence and requests for materials should be addressed to J.N.S.

Reprints and permission information is available at http://www.nature.com/ reprints

Publisher's note Springer Nature remains neutral with regard to jurisdictional claims in published maps and institutional affiliations.

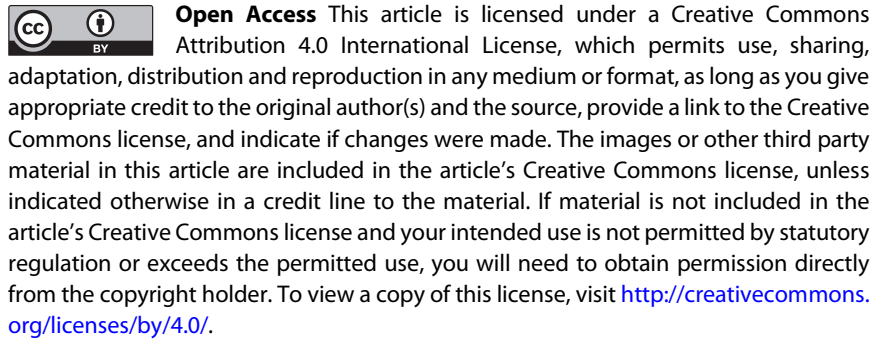

(c) The Author(s) 2021 